\title{
ON SIMPLE EIGENVALUES OF THE FRACTIONAL LAPLACIAN UNDER REMOVAL OF SMALL FRACTIONAL CAPACITY SETS
}

\author{
LAURA ABATANGELO, VERONICA FELLI, AND BENEDETTA NORIS
}

\begin{abstract}
We consider the eigenvalue problem for the restricted fractional Laplacian in a bounded domain with homogeneous Dirichlet boundary conditions. We introduce the notion of fractional capacity for compact subsets, with the property that the eigenvalues are not affected by the removal of zero fractional capacity sets. Given a simple eigenvalue, we remove from the domain a family of compact sets which are concentrating to a set of zero fractional capacity and we detect the asymptotic expansion of the eigenvalue variation; this expansion depends on the eigenfunction associated to the limit eigenvalue. Finally, we study the case in which the family of compact sets is concentrating to a point.
\end{abstract}

Keywords. Fractional Laplacian; Asymptotics of eigenvalues; Fractional capacity.

MSC classification. 31C15, 35P20, 35R11

\section{INTRODUCTION}

In the present paper we consider the eigenvalue problem for the Dirichlet fractional Laplacian in a bounded domain of $\mathbb{R}^{N}$. Our aim is to provide asymptotic estimates of the eigenvalue variation when a small vanishing set is removed. In this context, the good notion of smallness ensuring stability of the eigenvalue variation is related to the Gagliardo fractional capacity, which generalizes to the fractional setting the condenser capacity appearing in the framework of the standard Laplace operator, see Definition 1.1 below.

In the classical setting of the Dirichlet Laplacian, Rauch and Taylor [26] observed that the spectrum does not change by imposing homogeneous Dirichlet conditions on a compact polar subset, i.e. on a subset of zero Newtonian capacity. Courtois [13] developed a perturbation theory for the Dirichlet spectrum of a domain with small holes, with the capacity of holes playing the role of a perturbation parameter. More precisely, in [13] it is proved that, if $K \subset \Omega$ is a compact set, the $N$-th Dirichlet eigenvalue of the Laplacian in $\Omega \backslash K$ is close to the $N$-th Dirichlet eigenvalue of the Laplacian in $\Omega$ if (and only if) the capacity of the removed set $K$ in $\Omega$ is close to zero; furthermore, if the capacity of $K$ is small, then the eigenvalue variation is even differentiable with respect to the capacity of $K$ in $\Omega$. In [1] asymptotic estimates for such eigenvalue variation were obtained, highlighting a sharp relation between the order of vanishing of an eigenfunction of the Dirichlet Laplacian at a point and the leading term of the asymptotic expansion of the eigenvalue, as a removed compact set concentrates at that point. We also mention [4, 5, 12, 16, 25] for related estimates of the eigenvalue variation for the Laplacian under removal of small sets.

In order to formulate our problem, let us first introduce a suitable functional setting. Let $\Omega \subset \mathbb{R}^{N}, N \geq 1$, be an open set (bounded or unbounded). For $s \in(0, \min \{1, N / 2\})$, we define the homogeneous fractional Sobolev space $\mathcal{D}^{s, 2}(\Omega)$ as the completion of $C_{c}^{\infty}(\Omega)$ with respect to the Gagliardo norm

$$
[u]_{H^{s}\left(\mathbb{R}^{N}\right)}=\left(\int_{\mathbb{R}^{N}} \int_{\mathbb{R}^{N}} \frac{|u(x)-u(y)|^{2}}{|x-y|^{N+2 s}} d x d y\right)^{\frac{1}{2}} .
$$

Date: February 19, 2019.

L. Abatangelo and V. Felli are partially supported by the PRIN 2015 grant "Variational methods, with applications to problems in mathematical physics and geometry" and the INDAM-GNAMPA 2018 grant "Formula di monotonia e applicazioni: problemi frazionari e stabilità spettrale rispetto a perturbazioni del dominio". B. Noris is partially supported by the INDAM-GNAMPA group. All authors are partially supported by the project ERC Advanced Grant 2013 n. 339958: "Complex Patterns for Strongly Interacting Dynamical Systems - COMPAT" . 
We note that $\mathcal{D}^{s, 2}(\Omega) \hookrightarrow \mathcal{D}^{s, 2}\left(\mathbb{R}^{N}\right)$ continuously by trivial extension. $\mathcal{D}^{s, 2}(\Omega)$ is a Hilbert space with the scalar product

$$
(u, v)_{\mathcal{D}^{s, 2}(\Omega)}=\frac{C(N, s)}{2} \int_{\mathbb{R}^{N}} \int_{\mathbb{R}^{N}} \frac{(u(x)-u(y))(v(x)-v(y))}{|x-y|^{N+2 s}} d x d y=\int_{\mathbb{R}^{N}}|\xi|^{2 s} \overline{\widehat{v}(\xi)} \widehat{u}(\xi) d \xi,
$$

and the associated norm

$$
\|u\|_{\mathcal{D}^{s, 2}(\Omega)}=(u, u)_{\mathcal{D}^{s, 2}(\Omega)}^{1 / 2}=\sqrt{\frac{C(N, s)}{2}}[u]_{H^{s}\left(\mathbb{R}^{N}\right)},
$$

where

$$
C(N, s)=\pi^{-\frac{N}{2}} 2^{2 s} \frac{\Gamma\left(\frac{N+2 s}{2}\right)}{\Gamma(2-s)} s(1-s)
$$

$\Gamma$ is the Gamma function, and $\widehat{u}$ denotes the unitary Fourier transform of $u$.

We observe that, if $\Omega$ is bounded, then an equivalent norm on $\mathcal{D}^{s, 2}(\Omega)$ is

$$
\|u\|_{L^{2}(\Omega)}+[u]_{H^{s}\left(\mathbb{R}^{N}\right)},
$$

see [7, Corollary 5.2]. As observed in [8, 10, in general the space $\mathcal{D}^{s, 2}(\Omega)$ is smaller than the space $H_{0}^{s}(\Omega)$ defined as the closure of $C_{c}^{\infty}(\Omega)$ with respect to the norm

$$
\|u\|_{H^{s}(\Omega)}=\|u\|_{L^{2}(\Omega)}+[u]_{H^{s}(\Omega)}
$$

where

$$
[u]_{H^{s}(\Omega)}=\left(\int_{\Omega} \int_{\Omega} \frac{|u(x)-u(y)|^{2}}{|x-y|^{N+2 s}} d x d y\right)^{\frac{1}{2}} .
$$

The two spaces $\mathcal{D}^{s, 2}(\Omega)$ and $H_{0}^{s}(\Omega)$ coincide when $\Omega$ is a bounded Lipschitz open set and $s \neq 1 / 2$, see [8, Proposition B.1]. Furthermore, defining $H^{s}(\Omega)$ as the space $\left\{u \in L^{2}(\Omega):[u]_{H^{s}(\Omega)}<+\infty\right\}$ endowed with the norm $\|u\|_{H^{s}(\Omega)}=\|u\|_{L^{2}(\Omega)}+[u]_{H^{s}(\Omega)}$ and $\widetilde{H}^{s}(\Omega)$ as the space of $H^{s}\left(\mathbb{R}^{N}\right)$ functions that are zero in $\mathbb{R}^{N} \backslash \Omega$, it is known that, if $\Omega$ is bounded and Lipschitz, then

$$
H_{0}^{s}(\Omega)=\widetilde{H}^{s}(\Omega) \quad \text { if } s \neq \frac{1}{2}
$$

and

see [19, Corollary 1.4.4.5].

$$
H_{0}^{s}(\Omega)=\widetilde{H}^{s}(\Omega)=H^{s}(\Omega) \quad \text { if } s<\frac{1}{2},
$$

A key role in the perturbation theory we are going to develop for singularly perturbed fractional eigenvalue problems is played by the Gagliardo fractional capacity.

Definition 1.1. Let $\Omega \subset \mathbb{R}^{N}$ be a bounded open set. Let $K \subset \Omega$ be a compact set and let $\zeta_{K} \in C_{c}^{\infty}(\Omega)$ be such that $\zeta_{K}=1$ in a neighborhood of $K$. For every $s \in(0, \min \{1, N / 2\})$, we define the Gagliardo s-fractional capacity of $K$ in $\Omega$ as

$$
\operatorname{Cap}_{\Omega}^{s}(K)=\inf \left\{\|u\|_{\mathcal{D}^{s, 2}(\Omega)}^{2}: u \in \mathcal{D}^{s, 2}(\Omega) \text { and } u-\zeta_{K} \in \mathcal{D}^{s, 2}(\Omega \backslash K)\right\} .
$$

The Gagliardo $s$-capacity was introduced and studied in several recent papers. We refer e.g. to [27, Appendix A] for some basic properties of the $s$-capacity; we also mention [2, 3, 30, 32] for some related notions of fractional capacity.

From now on $\Omega \subset \mathbb{R}^{N}$ will denote a bounded open set. We consider the following eigenvalue problem with homogeneous Dirichlet boundary conditions for the restricted fractional Laplacian:

$$
\begin{cases}(-\Delta)^{s} u=\lambda u, & \text { in } \Omega, \\ u=0, & \text { in } \mathbb{R}^{N} \backslash \Omega .\end{cases}
$$

We refer to Section 2 for a quick review of the definition and main properties of the fractional Laplacian $(-\Delta)^{s}$. We say that $\lambda \in \mathbb{R}$ is an eigenvalue of problem (1.3) if there exists some $u \in \mathcal{D}^{s, 2}(\Omega) \backslash\{0\}$ (called eigenfunction) such that

$$
(u, v)_{\mathcal{D}^{s, 2}(\Omega)}=\lambda \int_{\mathbb{R}^{N}} u(x) v(x) d x, \quad \text { for all } v \in \mathcal{D}^{s, 2}(\Omega) .
$$


Since $(-\Delta)^{s}$ is a self-adjoint operator on $L^{2}(\Omega)$ with compact inverse, the Spectral Theorem implies that the eigenvalues have finite multiplicity and form a diverging sequence

$$
0<\lambda_{1}^{s}(\Omega) \leq \lambda_{2}^{s}(\Omega) \leq \lambda_{3}^{s}(\Omega) \leq \ldots \rightarrow+\infty .
$$

We notice that, in contrast with the local case, a connectedness assumption on the domain $\Omega$ would lead to some loss of generality. Indeed, in the classical case the spectrum of the Dirichlet Laplacian in a disconnected domain is the union of the spectra on the connected components, whereas in the fractional case the spectrum is influenced by the mutual position of the connected components due to the nonlocal effects, see [9, §2.3].

We shall consider the eigenfunctions normalized as follows

$$
\int_{\Omega}\left|u_{j}(x)\right|^{2} d x=1 .
$$

Our first result is the fractional counterpart of [13, Theorem 1.1] and establishes the continuity of the eigenvalue variation under the removal of small fractional capacity sets.

Theorem 1.2. Let $\Omega \subset \mathbb{R}^{N}$ be a bounded open set. For $s \in(0, \min \{1, N / 2\}), K \subset \Omega$ compact and $k \in \mathbb{N}_{*}$, let $\lambda_{k}^{s}(\Omega)$, respectively $\lambda_{k}^{s}(\Omega \backslash K)$, be the $k$-th eigenvalue of problem (1.3) in $\Omega$, respectively $\Omega \backslash K$. There exist $C>0$ and $\delta>0$ (independent of $K$ ) such that, if $\operatorname{Cap}_{\Omega}^{s}(K) \leq \delta$, then

$$
0 \leq \lambda_{k}^{s}(\Omega \backslash K)-\lambda_{k}^{s}(\Omega) \leq C\left(\operatorname{Cap}_{\Omega}^{s}(K)\right)^{1 / 2} .
$$

In particular we have that $\lambda_{k}^{s}(\Omega \backslash K) \rightarrow \lambda_{k}^{s}(\Omega)$ as $\operatorname{Cap}_{\Omega}^{s}(K) \rightarrow 0$.

Let us now consider a family of compact sets concentrating to a set of zero capacity with the goal of detecting the leading term of the asymptotic expansion of the eigenvalue variation.

Definition 1.3. Let $\Omega \subset \mathbb{R}^{N}$ be a bounded open set. Let $\left\{K_{\varepsilon}\right\}_{\varepsilon>0}$ be a family of compact sets contained in $\Omega$. We say that $K_{\varepsilon}$ is concentrating to a compact set $K \subset \Omega$ if for every open set $\omega$ such that $K \subset \omega \subseteq \Omega$ there exists $\varepsilon_{\omega}>0$ such that $K_{\varepsilon} \subset \omega$ for every $0<\varepsilon<\varepsilon_{\omega}$.

We note that the limit set $K$ appearing in the previous definition could be not unique. We comment on this definition in Appendix B, where in particular we discuss the relation between Definition 1.3 and the classical notion of convergence of sets in the sense of Mosco.

To state our main results in this direction, we need to introduce the notion of fractional $u$ capacity for a function $u \in \mathcal{D}^{s, 2}(\Omega)$.

Definition 1.4. Let $\Omega \subset \mathbb{R}^{N}$ be a bounded open set, $K \subset \Omega$ a compact set and $s \in(0, \min \{1, N / 2\})$. For every $u \in \mathcal{D}^{s, 2}(\Omega)$, we define the $s$-fractional $u$-capacity of $K$ in $\Omega$ as

$$
\operatorname{Cap}_{\Omega}^{s}(K, u)=\inf \left\{\|w\|_{\mathcal{D}^{s, 2}(\Omega)}^{2}: w \in \mathcal{D}^{s, 2}(\Omega) \text { and } w-u \in \mathcal{D}^{s, 2}(\Omega \backslash K)\right\} .
$$

More generally, we can define the fractional relative $u$-capacity for every function $u \in H_{\mathrm{loc}}^{s}(\Omega)$. Indeed, letting $\zeta_{K} \in C_{c}^{\infty}(\Omega)$ be as in Definition 1.1, we have that $\zeta_{K} u \in \mathcal{D}^{s, 2}(\Omega)$, so that we can define

$$
\operatorname{Cap}_{\Omega}^{s}(K, u)=\inf \left\{\|w\|_{\mathcal{D}^{s, 2}(\Omega)}^{2}: w \in \mathcal{D}^{s, 2}(\Omega) \text { and } w-\zeta_{K} u \in \mathcal{D}^{s, 2}(\Omega \backslash K)\right\} .
$$

The following theorem provides a sharp asymptotic expansion of the eigenvalue variation under removing of a family of compact sets concentrating to a zero fractional capacity set. In the classical setting of the Dirichlet Laplacian an analogous result can be found in [1, Theorem 1.4], see also the proof of [13, Theorem 1.2].

Theorem 1.5. Let $\Omega \subset \mathbb{R}^{N}$ be a bounded open set. For $s \in(0, \min \{1, N / 2\})$ and $j \in \mathbb{N}_{*}$, let $\lambda_{j}^{s}(\Omega)$ be the $j$-th eigenvalue of (1.3). Let $\left\{K_{\varepsilon}\right\}_{\varepsilon>0}$ be a family of compact sets contained in $\Omega$ concentrating to a compact set $K \subset \Omega$ in the sense of Definition 1.3. If

$$
\lambda_{j}^{s}(\Omega) \text { is simple and } \operatorname{Cap}_{\Omega}^{s}(K)=0
$$

then

$$
\lambda_{j}^{s}\left(\Omega \backslash K_{\varepsilon}\right)-\lambda_{j}^{s}(\Omega)=\operatorname{Cap}_{\Omega}^{s}\left(K_{\varepsilon}, u_{j}\right)+o\left(\operatorname{Cap}_{\Omega}^{s}\left(K_{\varepsilon}, u_{j}\right)\right),
$$

as $\varepsilon \rightarrow 0^{+}$, where $u_{j} \in \mathcal{D}^{s, 2}(\Omega)$ is an eigenfunction associated to $\lambda_{j}^{s}(\Omega)$ normalized as in (1.4). 
We can estimate the asymptotic behavior of the $s$-fractional $u_{j}$-capacity as the family of compact sets $K_{\varepsilon}$ concentrates to a point, by exploiting some of the results in 15 . Without loss of generality, we can assume that the limit point is the origin, hence in the following we suppose that $0 \in \Omega$, with $\Omega$ being a bounded open set in $\mathbb{R}^{N}$. We study the asymptotic behaviour of the quantity $\operatorname{Cap}_{\Omega}^{s}\left(K_{\varepsilon}, u_{j}\right)$ when $K_{\varepsilon}=\varepsilon K$ for a given compact set $K \subset \mathbb{R}^{N}$ and $\varepsilon \rightarrow 0^{+}$. We observe that the family of compact sets $\{\varepsilon K\}_{\varepsilon>0}$ concentrates (in the sense of Definition 1.3) to the singleton $\{0\}$, which has zero $s$-capacity in $\Omega$ (see Example 2.5 ahead).

For $s \in(0, \min \{1, N / 2\})$ and $j \in \mathbb{N}_{*}$, let $\lambda_{j}^{s}(\Omega)$ be the $j$-th eigenvalue of problem (1.3) and let $u_{j} \in \mathcal{D}^{s, 2}(\Omega)$ be an eigenfunction associated to $\lambda_{j}^{s}(\Omega)$ normalized as in (1.4). In view of [15], the asymptotic behavior of $u_{j}$ at 0 can be described in terms of the eigenvalues and the eigenfunctions of the following eigenvalue problem

$$
\begin{cases}-\operatorname{div}_{\mathbb{S}^{N}}\left(\theta_{N+1}^{1-2 s} \nabla_{\mathbb{S}^{N}} \psi\right)=\mu \theta_{N+1}^{1-2 s} \psi, & \text { in } \mathbb{S}_{+}^{N}, \\ -\lim _{\theta_{N+1} \rightarrow 0^{+}} \theta_{N+1}^{1-2 s} \nabla_{\mathbb{S}^{N}} \psi \cdot \mathbf{e}_{N+1}=0, & \text { on } \partial \mathbb{S}_{+}^{N},\end{cases}
$$

where $\mathbb{S}_{+}^{N}$ is the $N$-dimensional half-sphere

$$
\mathbb{S}_{+}^{N}=\left\{\left(\theta_{1}, \theta_{2}, \ldots, \theta_{N+1}\right) \in \mathbb{S}^{N}: \theta_{N+1}>0\right\}=\left\{\frac{z}{|z|}: z \in \mathbb{R}^{N+1}, z \cdot \mathbf{e}_{N+1}>0\right\},
$$

with $\mathbf{e}_{N+1}=(0, \ldots, 0,1) \in \mathbb{R}^{N+1}$. From classical spectral theory, problem (1.7) admits a diverging sequence of real eigenvalues with finite multiplicity

$$
\mu_{1}^{s} \leq \mu_{2}^{s} \leq \cdots \leq \mu_{k}^{s} \leq \cdots
$$

Moreover $\mu_{1}^{s}=0$ and it is simple, i.e. $\mu_{1}^{s}<\mu_{2}^{s}$. We note that, for $s=\frac{1}{2}$, by reflection eigenfunctions of (1.7) are spherical harmonics; then $\left\{\mu_{k}^{1 / 2}: k \geq 1\right\}=\{(N+k-2)(k-1): k \geq 1\}$ and eigenfunctions associated to the eigenvalue $(N+k-2)(k-1)$ are spherical harmonics of degree $k-1$.

From [15, Theorem 4.1 and Lemma 4.2] there exist $k_{0} \geq 1$ and $\psi \not \equiv 0$ eigenfunction of problem (1.7) associated to the eigenvalue $\mu_{k_{0}}^{s}$ such that, letting

$$
\gamma_{s}=-\frac{N-2 s}{2}+\sqrt{\left(\frac{N-2 s}{2}\right)^{2}+\mu_{k_{0}}^{s}}
$$

it holds

$$
\tilde{u}_{\varepsilon}(x):=\varepsilon^{-\gamma_{s}} u_{j}(\varepsilon x) \rightarrow \hat{\psi}(x) \quad \text { in } H^{s}\left(B_{R}^{\prime}\right) \quad \text { as } \varepsilon \rightarrow 0^{+},
$$

for every $R>0$, where $B_{R}^{\prime}=\left\{x \in \mathbb{R}^{N}:|x|<R\right\}$ and

$$
\hat{\psi}(x):=|x|^{\gamma_{s}} \psi\left(\frac{x}{|x|}, 0\right) .
$$

We note that $\hat{\psi} \not \equiv 0$, see Section 2.1

Theorem 1.6. Let $\Omega \subset \mathbb{R}^{N}$ be a bounded open set with $0 \in \Omega$ and $K \subset \Omega$ compact. For every $\varepsilon>0$ let $K_{\varepsilon}=\varepsilon K$. For $s \in(0, \min \{1, N / 2\})$ and $j \in \mathbb{N}_{*}$, let $\lambda_{j}^{s}(\Omega)$ be the $j$-th eigenvalue of problem (1.3) and let $u_{j} \in \mathcal{D}^{s, 2}(\Omega)$ be an eigenfunction associated to $\lambda_{j}^{s}(\Omega)$ normalized as in (1.4). Then, as $\varepsilon \rightarrow 0^{+}$, it holds

$$
\operatorname{Cap}_{\Omega}^{s}\left(K_{\varepsilon}, u_{j}\right)=\varepsilon^{N+2\left(\gamma_{s}-s\right)}\left\{\operatorname{Cap}_{\mathbb{R}^{N}}^{s}(K, \hat{\psi})+o(1)\right\},
$$

with $\gamma_{s}$ and $\hat{\psi}$ as in (1.8) and (1.10) respectively.

As a consequence of Theorems [1.5] and 1.6] we deduce the following.

Theorem 1.7. Let $\Omega \subset \mathbb{R}^{N}$ be a bounded open set with $0 \in \Omega$ and $K \subset \Omega$ compact. For every $\varepsilon>0$ let $K_{\varepsilon}=\varepsilon K$. For $s \in(0, \min \{1, N / 2\})$ and $j \in \mathbb{N}_{*}$, let $\lambda_{j}^{s}(\Omega)$ be the $j$-th eigenvalue of 
problem (1.3) and let $u_{j} \in \mathcal{D}^{s, 2}(\Omega)$ be an associated eigenfunction satisfying (1.4). If $\lambda_{j}^{s}(\Omega)$ is simple, then, as $\varepsilon \rightarrow 0^{+}$, it holds

$$
\lambda_{j}^{s}\left(\Omega \backslash K_{\varepsilon}\right)-\lambda_{j}^{s}(\Omega)=\varepsilon^{N+2\left(\gamma_{s}-s\right)} \operatorname{Cap}_{\mathbb{R}^{N}}^{s}(K, \hat{\psi})+o\left(\varepsilon^{N+2\left(\gamma_{s}-s\right)}\right),
$$

with $\gamma_{s}$ and $\hat{\psi}$ as in (1.8) and (1.10) respectively.

The asymptotic expansion (1.12) is sharp whenever $\operatorname{Cap}_{\mathbb{R}^{N}}^{s}(K, \hat{\psi}) \neq 0$, for example when $K$ has nonzero Lebesgue measure in $\mathbb{R}^{N}$, as observed in Corollary 1.8 below. We mention that the fractional capacity $\operatorname{Cap}_{\mathbb{R}^{N}}^{s}(K, \hat{\psi})$ on the whole $\mathbb{R}^{N}$ appearing in the leading term of the expansion (1.12) is related to the weighted capacity of $K$ in $\mathbb{R}^{N+1}$ with respect to the Muckenhoupt weight $|t|^{1-2 s}$, see Remark 2.8, we refer to [20, Chapter 2] for a discussion on the properties of such capacity.

Corollary 1.8. Under the same assumptions as in Theorem 1.7, suppose moreover that the $N$ dimensional Lebesgue measure of $K$ is strictly positive. Then

$$
\lim _{\varepsilon \rightarrow 0^{+}} \frac{\lambda_{j}^{s}\left(\Omega \backslash K_{\varepsilon}\right)-\lambda_{j}^{s}(\Omega)}{\varepsilon^{N+2\left(\gamma_{s}-s\right)}}=\operatorname{Cap}_{\mathbb{R}^{N}}^{s}(K, \hat{\psi})>0 .
$$

Remark 1.9. It is worth mentioning that in the literature, besides the notion of restricted fractional Laplacian treated in the present paper, also the so called spectral fractional Laplacian (defined as the power of $-\Delta$ obtained by using its spectral decomposition) is often taken into consideration. The restricted and the spectral fractional Laplacians on bounded domains are different operators, as observed in 24] and 29. The problem of spectral stability investigated in the present paper turns out to be much simpler for the spectral fractional Laplacian than for the restricted one, since the eigenvalues of the spectral fractional $s$-Laplacian are just the $s$-power of the eigenvalues of the classical Dirichlet Laplacian; hence the asymptotics of eigenvalues under removal of small sets can be easily deduced from the classical case treated in [1.

Denoting as $\left\{\lambda_{j}(\Omega)\right\}_{j=1}^{\infty}$ the eigenvalues the Laplacian in a bounded open set $\Omega \subset \mathbb{R}^{N}$ with homogeneous boundary conditions and by $\varphi_{j}$ the eigenfunction associated to $\lambda_{j}(\Omega)$ normalized with respect to the $L^{2}(\Omega)$-norm, the spectral fractional Laplacian with homogeneous Dirichlet boundary conditions can be defined, for all $s \in(0,1)$, as

$$
\left(-\Delta_{\text {spectral }}\right)^{s} u(x)=\sum_{j=1}^{+\infty}\left(\lambda_{j}(\Omega)\right)^{s}\left(\int_{\Omega} u \varphi_{j} d x\right) \varphi_{j}(x), \quad x \in \Omega .
$$

The eigenvalues and the eigenfunctions of $\left(-\Delta_{\text {spectral }}\right)^{s}$ are, respectively, $\nu_{j}^{s}(\Omega):=\left(\lambda_{j}(\Omega)\right)^{s}$ and $\varphi_{j}$. Then, from [1, Theorem 1.4] it follows easily that, if $\lambda_{j}(\Omega)$ is simple and $\left\{K_{\varepsilon}\right\}_{\varepsilon>0}$ is a family of compact sets contained in $\Omega$ concentrating to a null capacity compact set, then

$$
\nu_{j}^{s}\left(\Omega \backslash K_{\varepsilon}\right)-\nu_{j}^{s}(\Omega)=s\left(\lambda_{j}(\Omega)\right)^{s-1} \operatorname{Cap}_{\Omega}\left(K_{\varepsilon}, \varphi_{j}\right)+o\left(\operatorname{Cap}_{\Omega}\left(K_{\varepsilon}, \varphi_{j}\right)\right),
$$

as $\varepsilon \rightarrow 0^{+}$, where $\operatorname{Cap}_{\Omega}\left(K_{\varepsilon}, \varphi_{j}\right)=\inf \left\{\int_{\Omega}|\nabla f|^{2}: f \in H_{0}^{1}(\Omega)\right.$ and $\left.f-\varphi_{j} \in H_{0}^{1}\left(\Omega \backslash K_{\varepsilon}\right)\right\}$. Asymptotic expansions of $\operatorname{Cap}_{\Omega}\left(K_{\varepsilon}, \varphi_{j}\right)$ are obtained in [1] in several situations.

Comparing the above asymptotic expansion for the spectral fractional Laplacian with the expansion derived in Theorem 1.7, we note that only in the case of the restricted fractional Laplacian the vanishing order of the eigenvalue variation depends on the power $s$; hence the eigenvalues of the two operators exhibit quite different asymptotic behaviours under removal of small sets.

The paper is organized as follows. In Section 2 we collect some preliminary results. In Sections 3 and 4 we prove respectively Theorems 1.2 and 1.5. In Section 5 we present the proofs of Theorems 1.6. 1.7 and of Corollary 1.8. Finally, in Appendix $\$$ we prove an $L^{\infty}$ bound for eigenfunctions which is needed in Section 3 and in Appendix B we discuss the Definition 1.3 of concentrating compact sets.

\section{PRELiminaries}

In this section we recall some known facts and present some preliminary results. 
2.1. Restricted fractional Laplacian and Caffarelli-Silvestre extension. The fractional Laplacian $(-\Delta)^{s}$ can be defined over the space $C_{c}^{\infty}\left(\mathbb{R}^{N}\right)$ by the principal value integral

$$
(-\Delta)^{s} u(x)=C(N, s) \lim _{\varepsilon \rightarrow 0^{+}} \int_{\mathbb{R}^{N} \backslash B_{\varepsilon}(x)} \frac{u(x)-u(y)}{|x-y|^{N+2 s}} d y,
$$

where $C(N, s)$ is given in (1.2), or equivalently through the Fourier transform:

$$
\mathcal{F}\left((-\Delta)^{s} u\right)(\xi)=|\xi|^{2 s} \mathcal{F} u(\xi), \quad \xi \in \mathbb{R}^{N} .
$$

The scalar product of $\mathcal{D}^{s, 2}\left(\mathbb{R}^{N}\right)$ defined in (1.1) is naturally associated to $(-\Delta)^{s}$, in the sense that $(-\Delta)^{s}$ can be extended to a bounded linear operator from $\mathcal{D}^{s, 2}\left(\mathbb{R}^{N}\right)$ to its dual $\left(\mathcal{D}^{s, 2}\left(\mathbb{R}^{N}\right)\right)^{*}$, which actually coincides with the Riesz isomorphism of $\mathcal{D}^{s, 2}\left(\mathbb{R}^{N}\right)$ with respect to the scalar product (1.1), i.e.

$$
\left(\mathcal{D}^{s, 2}\left(\mathbb{R}^{N}\right)\right)^{*}\left\langle(-\Delta)^{s} u, v\right\rangle_{\mathcal{D}^{s, 2}\left(\mathbb{R}^{N}\right)}=(u, v)_{\mathcal{D}^{s, 2}\left(\mathbb{R}^{N}\right)}
$$

for all $u, v \in \mathcal{D}^{s, 2}\left(\mathbb{R}^{N}\right)$.

In 11] Caffarelli and Silvestre proved that $(-\Delta)^{s}$ can be realized as a Dirichlet-to-Neumann operator, i.e. as an operator mapping a Dirichlet boundary condition to a Neumann condition via an extension problem on the half space

$$
\mathbb{R}_{+}^{N+1}=\left\{(x, t) \in \mathbb{R}^{N+1}: x \in \mathbb{R}^{N}, t>0\right\} .
$$

For every $U, V \in C_{c}^{\infty}\left(\overline{\mathbb{R}_{+}^{N+1}}\right)$, let

$$
q(U, V)=\int_{\mathbb{R}_{+}^{N+1}} t^{1-2 s} \nabla U(x, t) \cdot \nabla V(x, t) d x d t .
$$

We define $\mathcal{D}^{1,2}\left(\mathbb{R}_{+}^{N+1} ; t^{1-2 s}\right)$ as the completion of $C_{c}^{\infty}\left(\overline{\mathbb{R}_{+}^{N+1}}\right)$ with respect to the norm

$$
\|U\|_{\mathcal{D}^{1,2}\left(\mathbb{R}_{+}^{N+1} ; t^{1-2 s}\right)}=\sqrt{q(U, U)}
$$

There exists a well-defined continuous trace map

$$
\operatorname{Tr}: \mathcal{D}^{1,2}\left(\mathbb{R}_{+}^{N+1} ; t^{1-2 s}\right) \rightarrow \mathcal{D}^{s, 2}\left(\mathbb{R}^{N}\right)
$$

which is onto (see for example [6]). By the Caffarelli-Silvestre extension theorem [1], given $u \in \mathcal{D}^{s, 2}\left(\mathbb{R}^{N}\right)$, the minimization problem

$$
\min \left\{q(W, W): W \in \mathcal{D}^{1,2}\left(\mathbb{R}_{+}^{N+1} ; t^{1-2 s}\right), \operatorname{Tr} W=u\right\}
$$

admits a unique minimizer $U=\mathcal{H}(u) \in \mathcal{D}^{1,2}\left(\mathbb{R}_{+}^{N+1} ; t^{1-2 s}\right)$, which moreover satisfies

$$
q(\mathcal{H}(u), W)=\kappa_{s}(u, \operatorname{Tr} W)_{\mathcal{D}^{s, 2}\left(\mathbb{R}^{N}\right)}, \quad \text { for all } \varphi \in \mathcal{D}^{1,2}\left(\mathbb{R}_{+}^{N+1} ; t^{1-2 s}\right),
$$

where

i.e. $U=\mathcal{H}(u)$ weakly solves

$$
\kappa_{s}=\frac{\Gamma(1-s)}{2^{2 s-1} \Gamma(s)}
$$

$$
\begin{cases}-\operatorname{div}\left(t^{1-2 s} \nabla U\right)=0, & \text { in } \mathbb{R}_{+}^{N+1}, \\ \lim _{t \rightarrow 0^{+}}\left(-t^{1-2 s} \partial_{t} U\right)=\kappa_{s}(-\Delta)^{s} u, & \text { in } \mathbb{R}^{N} \times\{0\} .\end{cases}
$$

From (2.2) it follows that

$$
\|U\|_{\mathcal{D}^{1,2}\left(\mathbb{R}_{+}^{N+1} ; t^{1-2 s}\right)}^{2}=\kappa_{s}\|u\|_{\mathcal{D}^{s, 2}\left(\mathbb{R}^{N}\right)}^{2} .
$$

As a consequence, if $\lambda_{j}^{s}(\Omega)$ is an eigenvalue of (1.3) for a certain $j \in \mathbb{N}_{*}=\mathbb{N} \backslash\{0\}$ and $u_{j} \in \mathcal{D}^{s, 2}(\Omega)$ is an associated eigenfunction, the extension $U_{j}=\mathcal{H}\left(u_{j}\right)$ satisfies $\operatorname{Tr} U_{j}=u_{j}$ and

$$
\begin{cases}-\operatorname{div}\left(t^{1-2 s} \nabla U_{j}\right)=0, & \text { in } \mathbb{R}_{+}^{N+1}, \\ \lim _{t \rightarrow 0^{+}}\left(-t^{1-2 s} \partial_{t} U_{j}\right)=\lambda_{j}^{s}(\Omega) \kappa_{s} \operatorname{Tr} U_{j}, & \text { in } \Omega \times\{0\}, \\ U_{j}=0, & \text { in }\left(\mathbb{R}^{N} \backslash \Omega\right) \times\{0\},\end{cases}
$$


in a weak sense, that is

$$
\left\{\begin{array}{l}
U_{j} \in \mathcal{D}_{\Omega^{c}}^{1,2}\left(\mathbb{R}_{+}^{N+1} ; t^{1-2 s}\right), \\
q\left(U_{j}, \phi\right)=\lambda_{j}^{s}(\Omega) \kappa_{s} \int_{\Omega} \operatorname{Tr} U_{j} \operatorname{Tr} \phi d x \quad \text { for every } \phi \in \mathcal{D}_{\Omega^{c}}^{1,2}\left(\mathbb{R}_{+}^{N+1} ; t^{1-2 s}\right) .
\end{array}\right.
$$

Here, the space $\mathcal{D}_{\Omega^{c}}^{1,2}\left(\mathbb{R}_{+}^{N+1} ; t^{1-2 s}\right)$ is defined as the closure of $C_{c}^{\infty}\left(\mathbb{R}_{+}^{N+1} \cup \Omega\right)$ in $\mathcal{D}^{1,2}\left(\mathbb{R}_{+}^{N+1} ; t^{1-2 s}\right)$; we also have the equivalent characterization

$$
\mathcal{D}_{\Omega^{c}}^{1,2}\left(\mathbb{R}_{+}^{N+1} ; t^{1-2 s}\right)=\left\{U \in \mathcal{D}^{1,2}\left(\mathbb{R}_{+}^{N+1} ; t^{1-2 s}\right): \operatorname{Tr} U \in \mathcal{D}^{s, 2}(\Omega)\right\} .
$$

We can consider equivalently either (2.5) or (1.3) with $\lambda=\lambda_{j}^{s}(\Omega)$. In this extended setting, the eigenvalues admit the following Courant-Fisher minimax characterization

$$
\lambda_{j}^{s}(\Omega)=\min _{\mathcal{U} \in \mathcal{S}_{j}} \max _{\substack{U \in \mathcal{U} \\\|\operatorname{Tr} U\|_{L^{2}(\Omega)} \neq 0}} \mathcal{R}(U)
$$

where $\mathcal{S}_{j}$ denotes the family of all $j$-dimensional subspaces of $\mathcal{D}_{\Omega^{c}}^{1,2}\left(\mathbb{R}_{+}^{N+1} ; t^{1-2 s}\right)$ and $\mathcal{R}$ is the Rayleigh type quotient defined as

$$
\mathcal{R}(U)=\frac{q(U, U)}{\kappa_{s} \int_{\Omega}|\operatorname{Tr} U(x)|^{2} d x} .
$$

Remark 2.1. If $\Omega \subset \mathbb{R}^{N}$ is bounded and open and $K \subset \Omega$ is a compact subset, in view of the Caffarelli-Silvestre extension result described above and, in particular, of (2.3) , we can characterize the Gagliardo $s$-fractional capacity introduced in Definition 1.1 as

$$
\operatorname{Cap}_{\Omega}^{s}(K)=\frac{1}{\kappa_{s}} \inf \left\{q(W, W): W \in \mathcal{D}_{\Omega^{c}}^{1,2}\left(\mathbb{R}_{+}^{N+1} ; t^{1-2 s}\right) \text { and } W-\eta_{K} \in \mathcal{D}_{\Omega^{c} \cup K}^{1,2}\left(\mathbb{R}_{+}^{N+1} ; t^{1-2 s}\right)\right\},
$$

where $\eta_{K} \in C_{c}^{\infty}\left(\mathbb{R}_{+}^{N+1} \cup \Omega\right)$ is any fixed function such that $\eta_{K}=1$ in a neighborhood of $K$.

Correspondingly, for any $u \in \mathcal{D}^{s, 2}(\Omega)$, we can characterize the $s$-fractional $u$-capacity of $K$ in $\Omega$ introduced in Definition 1.4 as

$$
\operatorname{Cap}_{\Omega}^{s}(K, u)=\frac{1}{\kappa_{s}} \inf \left\{q(W, W): W \in \mathcal{D}_{\Omega^{c}}^{1,2}\left(\mathbb{R}_{+}^{N+1} ; t^{1-2 s}\right), W-U \in \mathcal{D}_{\Omega^{c} \cup K}^{1,2}\left(\mathbb{R}_{+}^{N+1} ; t^{1-2 s}\right)\right\}
$$

where $U \in \mathcal{D}_{\Omega^{c}}^{1,2}\left(\mathbb{R}_{+}^{N+1} ; t^{1-2 s}\right)$ is such that $\operatorname{Tr} U=u$.

2.2. Local asymptotic behaviour of eigenfunctions and their extension. For $j \in \mathbb{N}_{*}$ and $s \in\left(0, \min \left\{1, \frac{N}{2}\right\}\right)$, let $\lambda_{j}^{s}(\Omega)$ be the $j$-th eigenvalue of problem $(1.3)$ and let $U_{j} \in \mathcal{D}_{\Omega^{c}}^{1,2}\left(\mathbb{R}_{+}^{N+1} ; t^{1-2 s}\right)$ be a solution to (2.4) such that its trace $u_{j}=\operatorname{Tr} U_{j}$ satisfies the normalization condition (1.4). In [15, the asymptotic behavior of $U_{j}$ (and consequently of its trace $u_{j}$ ) at 0 has been described in terms of the eigenvalues and the eigenfunctions of problem (1.7). More precisely, in [15, Theorem 4.1 and Lemma 4.2] it has been proved that there exist $k_{0} \geq 1$ and $\psi \not \equiv 0$ eigenfunction of problem (1.7) associated to the eigenvalue $\mu_{k_{0}}^{s}$ such that

$$
\tilde{U}_{\varepsilon}(z):=\varepsilon^{-\gamma_{s}} U_{j}(\varepsilon z) \rightarrow \tilde{\psi}(z):=|z|^{\gamma_{s}} \psi\left(\frac{z}{|z|}\right) \quad \text { in } H^{1}\left(B_{R}^{+} ; t^{1-2 s}\right) \quad \text { as } \varepsilon \rightarrow 0^{+},
$$

for every $R>0$, where $B_{R}^{+}=\left\{z=(x, t) \in \mathbb{R}_{+}^{N+1}:|z|<R\right\}, \gamma_{s}$ is given in (1.8), and the space $H^{1}\left(B_{R}^{+} ; t^{1-2 s}\right)$ is defined in Section 2.3 below.

The convergence (1.9) stated in the introduction follows from (2.10) by passing to the traces.

Remark 2.2. We note that the limit profile $\hat{\psi}:=\operatorname{Tr} \tilde{\psi}$ appearing in (1.9) is not identically null; indeed $\tilde{\psi}$ and $t^{1-2 s} \partial_{t} \tilde{\psi}$ can not both vanish on $\partial \mathbb{R}_{+}^{N+1}$, because otherwise $\tilde{\psi}$ would be a weak solution to the equation $\operatorname{div}\left(t^{1-2 s} \nabla \tilde{\psi}\right)=0$ satisfying both Dirichlet and weighted Neumann homogeneous boundary conditions and its trivial extension in $\mathbb{R}^{N+1}$ would violate the unique continuation principle for elliptic equations with Muckenhoupt weights proved in 31] (see also [18, and [28, Proposition 2.2]). 
2.3. Sobolev and Hardy-type inequalities. For every $s \in\left(0, \min \left\{1, \frac{N}{2}\right\}\right)$ (so that $\left.N-2 s>0\right)$, let

$$
2^{*}(s)=\frac{2 N}{N-2 s} .
$$

The following Sobolev inequalities and compactness results can be found for example in [14].

Theorem 2.3 (14, Theorems 6.5 and 6.7, Corollary 7.2]). Let $\Omega \subset \mathbb{R}^{N}, N \geq 1$, be a bounded, open set of class $C^{0,1}$ and let $s \in(0, \min \{1, N / 2\})$.

(i) There exists a positive constant $S_{N, s}$ such that

$$
S_{N, s}\|u\|_{L^{2^{*}(s)}\left(\mathbb{R}^{N}\right)} \leq\|u\|_{\mathcal{D}^{s, 2}\left(\mathbb{R}^{N}\right)} \quad \text { for all } u \in \mathcal{D}^{s, 2}\left(\mathbb{R}^{N}\right) .
$$

(ii) There exists a positive constant $C=C(N, s, \Omega)$ such that for every $u \in H^{s}(\Omega)$ and for every $q \in\left[1,2^{*}(s)\right]$ it holds

$$
\|u\|_{L^{q}(\Omega)} \leq C\|u\|_{H^{s}(\Omega)} .
$$

(iii) If $\mathcal{I}$ is a bounded subset of $H^{s}(\Omega)$, then $\mathcal{I}$ is pre-compact in $L^{q}(\Omega)$ for every $q \in\left[1,2^{*}(s)\right)$.

Let us recall some fractional Hardy-type inequalities. For any $s \in(0,1)$, the following Hardytype inequality for $\mathcal{D}^{s, 2}\left(\mathbb{R}^{N}\right)$-functions was established in [22]:

$$
\Lambda_{N, s} \int_{\mathbb{R}^{N}} \frac{u^{2}(x)}{|x|^{2 s}} d x \leq\|u\|_{\mathcal{D}^{s, 2}\left(\mathbb{R}^{N}\right)}^{2} \quad \text { for all } u \in \mathcal{D}^{s, 2}\left(\mathbb{R}^{N}\right),
$$

where

$$
\Lambda_{N, s}=2^{2 s} \frac{\Gamma^{2}\left(\frac{N+2 s}{4}\right)}{\Gamma^{2}\left(\frac{N-2 s}{4}\right)} .
$$

By combining the (2.12) and (2.3), we obtain the following Hardy-trace inequality:

$$
\Lambda_{N, s} \kappa_{s} \int_{\mathbb{R}^{N}} \frac{|\operatorname{Tr} U|^{2}}{|x|^{2 s}} d x \leq \int_{\mathbb{R}_{+}^{N+1}} t^{1-2 s}|\nabla U|^{2} d x d t, \quad \text { for all } U \in \mathcal{D}^{1,2}\left(\mathbb{R}_{+}^{N+1} ; t^{1-2 s}\right) .
$$

Relation (2.13) implies in particular that, if $\Omega$ is bounded,

$$
\int_{\Omega}|\operatorname{Tr} U|^{2} d x \leq \frac{\operatorname{diam}(\Omega)^{2 s}}{\Lambda_{N, s} \kappa_{s}}\|U\|_{\mathcal{D}^{1,2}\left(\mathbb{R}_{+}^{N+1} ; t^{1-2 s}\right)}^{2}, \quad \text { for all } U \in \mathcal{D}_{\Omega^{c}}^{1,2}\left(\mathbb{R}_{+}^{N+1} ; t^{1-2 s}\right),
$$

where $\operatorname{diam}(\Omega)$ is the diameter of $\Omega$.

For $r>0$, let $B_{r}^{+}=\left\{z=(x, t) \in \mathbb{R}_{+}^{N+1}:|z|<r\right\}$. We define $H^{1}\left(B_{r}^{+} ; t^{1-2 s}\right)$ as the completion of $C^{\infty}\left(\overline{B_{r}^{+}}\right)$with respect to

$$
\|U\|_{H^{1}\left(B_{r}^{+} ; t^{1-2 s}\right)}=\left(\int_{B_{r}^{+}} t^{1-2 s}\left(|\nabla U|^{2}+U^{2}\right) d x d t\right)^{1 / 2} .
$$

The following Hardy type inequality with boundary terms was proved in [15].

Lemma 2.4 ([15, Lemma 2.4]). Let $s \in(0, \min \{1, N / 2\})$. For all $r>0$ and $U \in H^{1}\left(B_{r}^{+} ; t^{1-2 s}\right)$, the following holds

$$
\left(\frac{N-2 s}{2}\right)^{2} \int_{B_{r}^{+}} t^{1-2 s} \frac{U^{2}(z)}{|z|^{2}} d z \leq \int_{B_{r}^{+}} t^{1-2 s}\left(\nabla U(z) \cdot \frac{z}{|z|}\right)^{2} d z+\left(\frac{N-2 s}{2 r}\right) \int_{S_{r}^{+}} t^{1-2 s} U^{2}(z) d S,
$$

where $S_{r}^{+}=\left\{z=(x, t) \in \mathbb{R}_{+}^{N+1}:|z|=r\right\}$ and $d S$ denotes the volume element on $S_{r}^{+}$.

As a particular case of the inequality stated in Lemma 2.4, we obtain the following

$$
\left(\frac{N-2 s}{2}\right)^{2} \int_{\mathbb{R}_{+}^{N+1}} t^{1-2 s} \frac{U^{2}(z)}{|z|^{2}} d z \leq \int_{\mathbb{R}_{+}^{N+1}} t^{1-2 s}|\nabla U(z)|^{2} d z,
$$

for all $U \in \mathcal{D}^{1,2}\left(\mathbb{R}_{+}^{N+1} ; t^{1-2 s}\right)$ and $s \in(0, \min \{1, N / 2\})$. 
2.4. Fractional capacities and capacitary potentials. We observe that, by Stampacchia's Theorem, the infimum in Remark 2.1 is achieved by a unique function $V_{\Omega, K} \in \mathcal{D}_{\Omega^{c}}^{1,2}\left(\mathbb{R}_{+}^{N+1} ; t^{1-2 s}\right)$, with $V_{\Omega, K}-\eta_{K} \in \mathcal{D}_{\Omega^{c} \cup K}^{1,2}\left(\mathbb{R}_{+}^{N+1} ; t^{1-2 s}\right)$, so that

$$
\operatorname{Cap}_{\Omega}^{s}(K)=\frac{1}{\kappa_{s}} q\left(V_{\Omega, K}, V_{\Omega, K}\right)
$$

moreover $V_{\Omega, K}$ satisfies

$$
q\left(V_{\Omega, K}, v-V_{\Omega, K}\right) \geq 0
$$

for all $v \in \mathcal{D}_{\Omega^{c}}^{1,2}\left(\mathbb{R}_{+}^{N+1} ; t^{1-2 s}\right)$ with $v-\eta_{K} \in \mathcal{D}_{\Omega^{c} \cup K}^{1,2}\left(\mathbb{R}_{+}^{N+1} ; t^{1-2 s}\right)$. Equivalently, we have that $V_{\Omega, K} \in \mathcal{D}_{\Omega^{c}}^{1,2}\left(\mathbb{R}_{+}^{N+1} ; t^{1-2 s}\right)$ is the unique function such that $V_{\Omega, K}-\eta_{K} \in \mathcal{D}_{\Omega^{c} \cup K}^{1,2}\left(\mathbb{R}_{+}^{N+1} ; t^{1-2 s}\right)$ and

$$
q\left(V_{\Omega, K}, \phi\right)=0 \quad \text { for all } \phi \in \mathcal{D}_{\Omega^{c} \cup K}^{1,2}\left(\mathbb{R}_{+}^{N+1} ; t^{1-2 s}\right),
$$

that is to say, $V_{\Omega, K}$ is the unique weak solution of

$$
\begin{cases}-\operatorname{div}\left(t^{1-2 s} \nabla V_{\Omega, K}\right)=0, & \text { in } \mathbb{R}_{+}^{N+1}, \\ \lim _{t \rightarrow 0^{+}}\left(-t^{1-2 s} \partial_{t} V_{\Omega, K}\right)=0, & \text { in }(\Omega \backslash K) \times\{0\}, \\ V_{\Omega, K}=0, & \text { in }\left(\mathbb{R}^{N} \backslash \Omega\right) \times\{0\}, \\ V_{\Omega, K}=1, & \text { in } K \times\{0\} .\end{cases}
$$

We also observe that $\operatorname{Tr} V_{\Omega, K}$ attains the infimum in Definition 1.1

Since $V_{\Omega, K}^{-}$and $\left(V_{\Omega, K}-1\right)^{+}$belong to $\mathcal{D}_{\Omega^{c} \cup K}^{1,2}\left(\mathbb{R}_{+}^{N+1} ; t^{1-2 s}\right)$, we can choose $\phi=V_{\Omega, K}^{-}$and $\phi=\left(V_{\Omega, K}-1\right)^{+}$in (2.17); in this way we obtain that $V_{\Omega, K}^{-}=\left(V_{\Omega, K}-1\right)^{+} \equiv 0$, that is

$$
0 \leq V_{\Omega, K} \leq 1 \quad \text { a.e. in } \mathbb{R}_{+}^{N+1} \text {. }
$$

Example 2.5 (Capacity of a point). If $\Omega \subset \mathbb{R}^{N}$ is an open set, $s \in(0, \min \{1, N / 2\})$, and $P \in \Omega$, then

$$
\operatorname{Cap}_{\Omega}^{s}(\{P\})=0 .
$$

Indeed, for every $n \in \mathbb{N}_{*}$, let $W_{n} \in C^{\infty}\left(\mathbb{R}^{N+1}\right)$ be such that $W_{n}(z)=1$ for $|z-P| \leq \frac{1}{n}, W_{n}(z)=0$ for $|z-P| \geq \frac{2}{n}$, and $\left|\nabla W_{n}(z)\right| \leq 2 n$ for all $z \in \mathbb{R}^{N+1}$. Then, for $n$ sufficiently large, the restriction $\left.W_{n}\right|_{\mathbb{R}_{+}^{N+1}}$ belongs to $\mathcal{D}_{\Omega^{c}}^{1,2}\left(\mathbb{R}_{+}^{N+1} ; t^{1-2 s}\right)$ and is equal to 1 in a neighborhood of $\{P\}$. Moreover

$$
q\left(W_{n}, W_{n}\right) \leq \mathrm{const} n^{2} \int_{1 / n}^{2 / n} r^{N+1-2 s} d r=O\left(n^{2 s-N}\right)=o(1) \quad \text { as } n \rightarrow+\infty,
$$

thus proving (2.20).

In order to prove that the spectrum of restricted fractional $s$-Laplacian in $\Omega$ does not change by removing a subset of zero fractional $s$-capacity, the following result is needed.

Proposition 2.6. Let $\Omega \subset \mathbb{R}^{N}$ be an open set, $K \subset \Omega$ compact and $s \in(0, \min \{1, N / 2\})$. The following three assertions are equivalent:

(i) $\operatorname{Cap}_{\Omega}^{s}(K)=0$;

(ii) $\mathcal{D}_{\Omega^{c}}^{1,2}\left(\mathbb{R}_{+}^{N+1} ; t^{1-2 s}\right)=\mathcal{D}_{\Omega^{c} \cup K}^{1,2}\left(\mathbb{R}_{+}^{N+1} ; t^{1-2 s}\right)$;

(iii) $\mathcal{D}^{s, 2}(\Omega)=\mathcal{D}^{s, 2}(\Omega \backslash K)$.

Proof. It will be sufficient to prove that (i) is equivalent to (ii), since then the equivalence of (iii) follows from the fact that the restriction to $\Omega$ of the trace map Tr defined in (2.1) is onto and the characterization of spaces given in (2.6).

Suppose first that $\mathcal{D}_{\Omega^{c}}^{1,2}\left(\mathbb{R}_{+}^{N+1} ; t^{1-2 s}\right)=\mathcal{D}_{\Omega^{c} \cup K}^{1,2}\left(\mathbb{R}_{+}^{N+1} ; t^{1-2 s}\right)$. Then we can take $\phi=V_{\Omega, K}$ as a test function in (2.17), so that

$$
\operatorname{Cap}_{\Omega}^{s}(K)=q\left(V_{\Omega, K}, V_{\Omega, K}\right)=0 .
$$

Now suppose that $\operatorname{Cap}_{\Omega}^{s}(K)=0$. We have to show $\mathcal{D}_{\Omega^{c}}^{1,2}\left(\mathbb{R}_{+}^{N+1} ; t^{1-2 s}\right) \subset \mathcal{D}_{\Omega^{c} \cup K}^{1,2}\left(\mathbb{R}_{+}^{N+1} ; t^{1-2 s}\right)$, the other inclusion being evident. To this aim, let $u \in \mathcal{D}_{\Omega^{c}}^{1,2}\left(\mathbb{R}_{+}^{N+1} ; t^{1-2 s}\right)$. By the assumption that 
$\operatorname{Cap}_{\Omega}^{s}(K)=0$, for any $n \in \mathbb{N}$ there exists $\eta_{n} \in C_{c}^{\infty}\left(\mathbb{R}_{+}^{N+1} \cup \Omega\right)$ such that $\eta_{n} \equiv 1$ in a neighborhood of $K$ and

$$
\int_{\mathbb{R}_{+}^{N+1}} t^{1-2 s}\left|\nabla \eta_{n}\right|^{2} d x d t<\frac{1}{n}
$$

On the other hand, by density of $C_{c}^{\infty}\left(\mathbb{R}_{+}^{N+1} \cup \Omega\right)$ in $\mathcal{D}_{\Omega^{c}}^{1,2}\left(\mathbb{R}_{+}^{N+1} ; t^{1-2 s}\right)$, for any $\varepsilon>0$ there exists $u_{\varepsilon} \in C_{c}^{\infty}\left(\mathbb{R}_{+}^{N+1} \cup \Omega\right)$ such that

$$
\left\|u_{\varepsilon}-u\right\|_{\mathcal{D}^{1,2}\left(\mathbb{R}_{+}^{N+1} ; t^{1-2 s}\right)}^{2}<\varepsilon .
$$

In this way, the function $u_{\varepsilon}\left(1-\eta_{n}\right) \in C_{c}^{\infty}\left(\mathbb{R}_{+}^{N+1} \cup(\Omega \backslash K)\right)$; we estimate

$$
\begin{aligned}
\int_{\mathbb{R}_{+}^{N+1}} & t^{1-2 s}\left|\nabla\left(u_{\varepsilon}\left(1-\eta_{n}\right)-u\right)\right|^{2} d x d t=\int_{\mathbb{R}_{+}^{N+1}} t^{1-2 s}\left|\nabla u_{\varepsilon}-\nabla u-\nabla\left(\eta_{n} u_{\varepsilon}\right)\right|^{2} d x d t \\
& \leq 2 \int_{\mathbb{R}_{+}^{N+1}} t^{1-2 s}\left|\nabla u_{\varepsilon}-\nabla u\right|^{2} d x d t+2 \int_{\mathbb{R}_{+}^{N+1}} t^{1-2 s}\left|\nabla\left(\eta_{n} u_{\varepsilon}\right)\right|^{2} d x d t \\
& \leq 2 \varepsilon+4 \int_{\mathbb{R}_{+}^{N+1}} t^{1-2 s}\left|u_{\varepsilon}\right|^{2}\left|\nabla \eta_{n}\right|^{2} d x d t+4 \int_{\mathbb{R}_{+}^{N+1}} t^{1-2 s}\left|\eta_{n}\right|^{2}\left|\nabla u_{\varepsilon}\right|^{2} d x d t \\
& \leq 2 \varepsilon+\frac{4}{n} \sup \left|u_{\varepsilon}\right|^{2}+4\left(\sup \left|\nabla u_{\varepsilon}\right|^{2}\right) \int_{\operatorname{supp} u_{\varepsilon}} t^{1-2 s}\left|\eta_{n}\right|^{2} d x d t \\
& \leq 2 \varepsilon+\frac{4}{n} \sup \left|u_{\varepsilon}\right|^{2}+\frac{4}{n}\left(\frac{2}{N-2 s}\right)^{2} \sup \left|\nabla u_{\varepsilon}\right|^{2} \sup _{z \in \operatorname{supp} u_{\varepsilon}}|z|^{2}
\end{aligned}
$$

where the last relation relies on (2.15).

This proves that $u$ can be approximated in $\mathcal{D}^{1,2}\left(\mathbb{R}_{+}^{N+1} ; t^{1-2 s}\right)$ with $C_{c}^{\infty}\left(\mathbb{R}_{+}^{N+1} \cup(\Omega \backslash K)\right)$ functions, so that $u \in \mathcal{D}_{\Omega^{c} \cup K}^{1,2}\left(\mathbb{R}_{+}^{N+1} ; t^{1-2 s}\right)$.

As a direct consequence of Proposition 2.6. we obtain that the removal of a zero fractional $s$-capacity set leaves the family of eigenvalues of $(-\Delta)^{s}$ unchanged.

Corollary 2.7. Let $\Omega \subset \mathbb{R}^{N}$ be a bounded open set, $K \subset \Omega$ compact and $s \in(0, \min \{1, N / 2\})$. It holds $\lambda_{k}^{s}(\Omega)=\lambda_{k}^{s}(\Omega \backslash K)$ for every $k \in \mathbb{N}_{*}$ if and only if $\operatorname{Cap}_{\Omega}^{s}(K)=0$.

Proof. The result follows from Proposition 2.6 combined with (2.7) and the Spectral Theorem.

Remark 2.8. In the case $\Omega=\mathbb{R}^{N}$ and $K \subset \mathbb{R}^{N}$ compact, it holds

$$
2 \operatorname{Cap}_{\mathbb{R}^{N}}^{s}(K)=\operatorname{Cap}_{2,|t|^{1-2 s}}\left(K, \mathbb{R}^{N+1}\right),
$$

where the right hand side of the above expression is the $\left(2,|t|^{1-2 s}\right)$-capacity of the condenser $\left(K, \mathbb{R}^{N+1}\right)$, as introduced in [20, Chapter 2]. To see this, it suffices to consider the function $V_{K}:=V_{\mathbb{R}^{N}, K}$ that achieves $\operatorname{Cap}_{\mathbb{R}^{N}}^{s}(K)$ and its even extension

and to notice that

$$
\tilde{V}_{K}(x, t)= \begin{cases}V_{K}(x, t), & \text { if } t \geq 0, \\ V_{K}(x,-t), & \text { if } t<0,\end{cases}
$$

$$
\operatorname{Cap}_{\mathbb{R}^{N}}^{s}(K)=\frac{1}{2} \int_{\mathbb{R}^{N+1}}|t|^{1-2 s}\left|\nabla \tilde{V}_{K}\right|^{2} d x d t=\frac{1}{2} \operatorname{Cap}_{2,|t|^{1-2 s}}\left(K, \mathbb{R}^{N+1}\right) .
$$

We remark that $|t|^{1-2 s}$ is a 2-admissible weight (according to the definition given in 20, Chapter $2]$ ), since $|t|^{1-2 s}$ belongs to the Muckenhoupt class $A_{2}$.

Concerning the $s$-fractional $u$-capacity of $K$ in $\Omega$ introduced in Definition 1.4 and characterized equivalently in (2.9), we have that, as it happens for $\operatorname{Cap}_{\Omega}^{s}(K)$, the infimum in (2.9) is achieved by a function $V_{\Omega, K, u} \in \mathcal{D}_{\Omega^{c}}^{1,2}\left(\mathbb{R}_{+}^{N+1} ; t^{1-2 s}\right)$ and the infimum in (1.5) by $\operatorname{Tr} V_{\Omega, K, u}$, so that

$$
\operatorname{Cap}_{\Omega}^{s}(K, u)=\frac{1}{\kappa_{s}} q\left(V_{\Omega, K, u}, V_{\Omega, K, u}\right)=\left\|\operatorname{Tr} V_{\Omega, K, u}\right\|_{\mathcal{D}^{s, 2}(\Omega)}^{2},
$$


and $V_{\Omega, K, u}$ is the unique weak solution of

$$
\begin{cases}-\operatorname{div}\left(t^{1-2 s} \nabla V_{\Omega, K, u}\right)=0, & \text { in } \mathbb{R}_{+}^{N+1}, \\ \lim _{t \rightarrow 0^{+}}\left(-t^{1-2 s} \partial_{t} V_{\Omega, K, u}\right)=0, & \text { in }(\Omega \backslash K) \times\{0\}, \\ V_{\Omega, K, u}=0, & \text { in }\left(\mathbb{R}^{N} \backslash \Omega\right) \times\{0\}, \\ V_{\Omega, K, u}=u, & \text { in } K \times\{0\},\end{cases}
$$

in the sense that $V_{\Omega, K, u} \in \mathcal{D}_{\Omega^{c}}^{1,2}\left(\mathbb{R}_{+}^{N+1} ; t^{1-2 s}\right), V_{\Omega, K, u}-U \in \mathcal{D}_{\Omega^{c} \cup K}^{1,2}\left(\mathbb{R}_{+}^{N+1} ; t^{1-2 s}\right)$ for some function $U \in \mathcal{D}_{\Omega^{c}}^{1,2}\left(\mathbb{R}_{+}^{N+1} ; t^{1-2 s}\right)$ such that $\operatorname{Tr} U=u$, and

$$
q\left(V_{\Omega, K, u}, \phi\right)=0 \quad \text { for all } \phi \in \mathcal{D}_{\Omega^{c} \cup K}^{1,2}\left(\mathbb{R}_{+}^{N+1} ; t^{1-2 s}\right) .
$$

\section{Continuity of the eigenvalue variation}

Proof of Theorem 1.2. For every $j \in\{1,2, \ldots, k\}$, let $\lambda_{j}^{s}(\Omega)$ and $U_{j} \in \mathcal{D}_{\Omega^{c}}^{1,2}\left(\mathbb{R}_{+}^{N+1} ; t^{1-2 s}\right)$ solve (2.4) and (1.4). Moreover we can choose the eigenfunctions $U_{j}$ in such a way that

$$
\int_{\Omega} \operatorname{Tr} U_{j}(x) \operatorname{Tr} U_{\ell}(x) d x=0 \quad \text { for } j \neq \ell .
$$

Let us denote $u_{j}=\operatorname{Tr} U_{j}$ for all $j$. Let

$$
E=\operatorname{span}\left\{\Phi_{j}: j=1,2, \ldots, k\right\} \subset \mathcal{D}_{\Omega^{c} \cup K}^{1,2}\left(\mathbb{R}_{+}^{N+1} ; t^{1-2 s}\right)
$$

where $\Phi_{j}=U_{j}\left(1-V_{\Omega, K}\right)$ and $V_{\Omega, K}$ is the capacitary potential of $K$ satisfying (2.17)-(2.18). We denote $\varphi_{j}=\operatorname{Tr} \Phi_{j}$ for all $j$ and $v_{\Omega, K}=\operatorname{Tr} V_{\Omega, K}$. We observe that, in view of (1.4), (3.1), (2.14) and Lemma A.1, we have, for all $j, \ell \in\{1, \ldots, k\}$,

$$
\begin{aligned}
\left|\int_{\Omega} \varphi_{j}(x) \varphi_{\ell}(x) d x-\delta_{j \ell}\right| & =\left|-2 \int_{\Omega} u_{j}(x) u_{\ell}(x) v_{\Omega, K}(x) d x+\int_{\Omega} u_{j}(x) u_{\ell}(x) v_{\Omega, K}^{2}(x) d x\right| \\
& \leq\left(\max _{1 \leq j \leq k}\left\|u_{j}\right\|_{L^{\infty}(\Omega)}\right)^{2}\left(2 \int_{\Omega}\left|v_{\Omega, K}(x)\right| d x+\int_{\Omega} v_{\Omega, K}^{2}(x) d x\right) \\
& \leq C\left(\left(\operatorname{Cap}_{\Omega}^{s}(K)\right)^{1 / 2}+\operatorname{Cap}_{\Omega}^{s}(K)\right)
\end{aligned}
$$

for some constant $C>0$ independent of $K$. On the other hand

$$
\begin{aligned}
& q\left(\Phi_{j}, \Phi_{\ell}\right)=\int_{\mathbb{R}_{+}^{N+1}} t^{1-2 s}\left(1-V_{\Omega, K}\right)^{2} \nabla U_{j} \cdot \nabla U_{\ell} d x d t+\int_{\mathbb{R}_{+}^{N+1}} t^{1-2 s} U_{j} U_{\ell}\left|\nabla V_{\Omega, K}\right|^{2} d x d t \\
& -\int_{\mathbb{R}_{+}^{N+1}} t^{1-2 s} U_{j}\left(1-V_{\Omega, K}\right) \nabla U_{\ell} \cdot \nabla V_{\Omega, K} d x d t-\int_{\mathbb{R}_{+}^{N+1}} t^{1-2 s} U_{\ell}\left(1-V_{\Omega, K}\right) \nabla U_{j} \cdot \nabla V_{\Omega, K} d x d t .
\end{aligned}
$$

Choosing $\phi=U_{\ell}\left(1-V_{\Omega, K}\right)^{2}$ in (2.5) we obtain that

$$
\begin{aligned}
\int_{\mathbb{R}_{+}^{N+1}} t^{1-2 s}\left(1-V_{\Omega, K}\right)^{2} \nabla U_{j} \cdot \nabla U_{\ell} d x d t= & 2 \int_{\mathbb{R}_{+}^{N+1}} t^{1-2 s}\left(1-V_{\Omega, K}\right) U_{\ell} \nabla U_{j} \cdot \nabla V_{\Omega, K} d x d t \\
& +\kappa_{s} \lambda_{j}^{s}(\Omega) \int_{\Omega} \varphi_{j}(x) \varphi_{\ell}(x) d x
\end{aligned}
$$

hence, thanks to Lemma A.1 and (3.2), for every $j, \ell \in\{1,2, \ldots, k\}$,

$$
\begin{aligned}
& \left|\int_{\mathbb{R}_{+}^{N+1}} t^{1-2 s} \nabla \Phi_{j} \cdot \nabla \Phi_{\ell} d x d t-\kappa_{s} \lambda_{j}^{s}(\Omega) \delta_{j \ell}\right| \\
& =\mid \int_{\mathbb{R}_{+}^{N+1}} t^{1-2 s}\left(1-V_{\Omega, K}\right) U_{\ell} \nabla U_{j} \cdot \nabla V_{\Omega, K} d x d t+\kappa_{s} \lambda_{j}^{s}(\Omega)\left(\int_{\Omega} \varphi_{j}(x) \varphi_{\ell}(x) d x-\delta_{j \ell}\right) \\
& \quad \quad+\int_{\mathbb{R}_{+}^{N+1}} t^{1-2 s} U_{j} U_{\ell}\left|\nabla V_{\Omega, K}\right|^{2} d x d t-\int_{\mathbb{R}_{+}^{N+1}} t^{1-2 s} U_{j}\left(1-V_{\Omega, K}\right) \nabla U_{\ell} \cdot \nabla V_{\Omega, K} d x d t \mid \\
& \quad \leq C\left(\left(\operatorname{Cap}_{\Omega}^{s}(K)\right)^{1 / 2}+\operatorname{Cap}_{\Omega}^{s}(K)\right)
\end{aligned}
$$


for some constant $C>0$ independent of $K$. The above estimate implies there exists $\delta>0$ independent of $K$ such that $\Phi_{1}, \Phi_{2}, \ldots, \Phi_{k}$ are linearly independent provided $\operatorname{Cap}_{\Omega}^{s}(K)<\delta$, so that $E$ is a $k$-dimensional subspace of $\mathcal{D}_{\Omega^{c} \cup K}^{1,2}\left(\mathbb{R}_{+}^{N+1} ; t^{1-2 s}\right)$ for $\operatorname{Cap}_{\Omega}^{s}(K)<\delta$.

From (2.7), the fact that $\lambda_{i}^{s}(\Omega) \leq \lambda_{k}^{s}(\Omega)$ for all $i \leq k$, (3.2) and (3.3) we have that

$$
\begin{aligned}
\lambda_{k}^{s}(\Omega \backslash K) & \leq \max _{\substack{\left(\alpha_{1}, \alpha_{2}, \ldots, \alpha_{k}\right) \in \mathbb{R}^{k} \\
\sum_{i=1}^{k} \alpha_{i}^{2}=1}} \mathcal{R}\left(\sum_{i=1}^{k} \alpha_{i} \Phi_{i}\right) \\
& =\max _{\substack{\left(\alpha_{1}, \alpha_{2}, \ldots, \alpha_{k}\right) \in \mathbb{R}^{k} \\
\sum_{i=1}^{k} \alpha_{i}^{2}=1}} \frac{\sum_{i, j=1}^{k} \alpha_{i} \alpha_{j} q\left(\Phi_{i}, \Phi_{j}\right)}{\kappa_{s} \sum_{i, j=1}^{k} \alpha_{i} \alpha_{j} \int_{\Omega} \varphi_{i} \varphi_{j} d x} \\
& =\max _{\substack{\left(\alpha_{1}, \alpha_{2}, \ldots, \alpha_{k}\right) \in \mathbb{R}^{k} \\
\sum_{i=1}^{k} \alpha_{i}^{2}=1}} \frac{\left(\sum_{i=1}^{k} \alpha_{i}^{2} \kappa_{s} \lambda_{i}^{s}(\Omega)\right)+O\left(\left(\operatorname{Cap}_{\Omega}^{s}(K)\right)^{1 / 2}\right)}{\kappa_{s}\left(1+O\left(\left(\operatorname{Cap}_{\Omega}^{s}(K)\right)^{1 / 2}\right)\right)} \\
& \leq \frac{\kappa_{s} \lambda_{k}^{s}(\Omega)+O\left(\left(\operatorname{Cap}_{\Omega}^{s}(K)\right)^{1 / 2}\right)}{\kappa_{s}\left(1+O\left(\left(\operatorname{Cap}_{\Omega}^{s}(K)\right)^{1 / 2}\right)\right)}=\lambda_{k}^{s}(\Omega)+O\left(\left(\operatorname{Cap}_{\Omega}^{s}(K)\right)^{1 / 2}\right)
\end{aligned}
$$

as $\operatorname{Cap}_{\Omega}^{s}(K) \rightarrow 0$. The proof is thereby complete.

\section{Asymptotic expansion of the Eigenvalues under Removal of SMAll CAPACity SETS}

The aim of this section is to prove Theorem 1.5. The proof is inspired from that of 1, Theorem 1.4]. Let us start with some preliminary lemmas concerning the capacitary potential $V_{\Omega, K, u}$ defined in (2.21)-2.22).

Lemma 4.1. Let $\left\{K_{\varepsilon}\right\}_{\varepsilon>0}$ be a family of compact sets contained in the open set $\Omega$ concentrating, in the sense of Definition 1.3, to a compact set $K \subset \Omega$, with $\operatorname{Cap}_{\Omega}^{s}(K)=0$. For every $u \in \mathcal{D}^{s, 2}(\Omega)$ it holds

$$
\int_{\Omega}\left|\operatorname{Tr} V_{\Omega, K_{\varepsilon}, u}\right|^{2} d x=o\left(\operatorname{Cap}_{\Omega}^{s}\left(K_{\varepsilon}, u\right)\right) \quad \text { as } \varepsilon \rightarrow 0 .
$$

Proof. Let $\mathcal{H}_{\varepsilon}=\mathcal{D}_{\Omega^{c} \cup K_{\varepsilon}}^{1,2}\left(\mathbb{R}_{+}^{N+1} ; t^{1-2 s}\right)$. Suppose by contradiction that there exist a sequence $\varepsilon_{n} \rightarrow 0, \varepsilon_{n}>0$, and a constant $C>0$ such that

$$
\int_{\Omega}\left|\operatorname{Tr} V_{\Omega, K_{\varepsilon_{n}}, u}\right|^{2} d x \geq C\left\|V_{\Omega, K_{\varepsilon_{n}}, u}\right\|_{\mathcal{D}_{\Omega^{c}}^{1,2}\left(\mathbb{R}_{+}^{N+1} ; t^{1-2 s}\right)}^{2}
$$

for every $n$. Letting

we have

$$
W_{n}=\frac{V_{\Omega, K_{\varepsilon_{n}}, u}}{\left\|\operatorname{Tr} V_{K_{\Omega, K_{\varepsilon_{n}}, u}}\right\|_{L^{2}(\Omega)}}
$$

$$
\left\|\operatorname{Tr} W_{n}\right\|_{L^{2}(\Omega)}=1 \quad \text { and } \quad\left\|W_{n}\right\|_{\mathcal{D}_{\Omega^{c}}^{1,2}\left(\mathbb{R}_{+}^{N+1} ; t^{1-2 s}\right)}^{2} \leq C^{-1}
$$

for every $n$. By weak compactness of the unit ball of $\mathcal{D}_{\Omega^{c}}^{1,2}\left(\mathbb{R}_{+}^{N+1} ; t^{1-2 s}\right)$ and by compactness of the trace operator $\operatorname{Tr}: \mathcal{D}_{\Omega^{c}}^{1,2}\left(\mathbb{R}_{+}^{N+1} ; t^{1-2 s}\right) \rightarrow L^{2}(\Omega)$ (which follows easily by combining the continuity of the trace map $\operatorname{Tr}: \mathcal{D}_{\Omega^{c}}^{1,2}\left(\mathbb{R}_{+}^{N+1} ; t^{1-2 s}\right) \rightarrow \mathcal{D}^{s, 2}(\Omega)$ and part (iii) of Theorem 2.3 ), there exist a subsequence $\left(n_{k}\right)_{k \geq 1}$ and $W \in \mathcal{D}_{\Omega^{c}}^{1,2}\left(\mathbb{R}_{+}^{N+1} ; t^{1-2 s}\right)$ such that

$$
W_{n_{k}} \rightarrow W \quad \text { in } \mathcal{D}_{\Omega^{c}}^{1,2}\left(\mathbb{R}_{+}^{N+1} ; t^{1-2 s}\right) \text { as } k \rightarrow+\infty \quad \text { and } \quad\|\operatorname{Tr} W\|_{L^{2}(\Omega)}=1 .
$$

Moreover, from (2.23) we deduce that

$$
\int_{\mathbb{R}_{+}^{N+1}} t^{1-2 s} \nabla W_{n_{k}} \cdot \nabla \phi d x d t=0 \quad \text { for every } \phi \in \mathcal{H}_{\varepsilon_{n_{k}}} .
$$


For every $\phi \in C_{c}^{\infty}\left(\mathbb{R}_{+}^{N+1} \cup(\Omega \backslash K)\right)$, we have that $\phi \in \mathcal{H}_{\varepsilon}$ for $\varepsilon$ sufficiently small. Therefore we can pass to the limit as $k \rightarrow+\infty$ above and obtain

$$
\int_{\mathbb{R}_{+}^{N+1}} t^{1-2 s} \nabla W \cdot \nabla \phi d x d t=0 \quad \text { for every } \phi \in C_{c}^{\infty}\left(\mathbb{R}_{+}^{N+1} \cup(\Omega \backslash K)\right) .
$$

By density, the latter holds for every $\phi \in \mathcal{D}_{\Omega^{c} \cup K}^{1,2}\left(\mathbb{R}_{+}^{N+1} ; t^{1-2 s}\right)$. Now, the assumption $\operatorname{Cap}_{\Omega}^{s}(K)=0$ allows to deduce, through Proposition 2.6.

$$
\int_{\mathbb{R}_{+}^{N+1}} t^{1-2 s} \nabla W \cdot \nabla \phi d x d t=0 \quad \text { for every } \phi \in \mathcal{D}_{\Omega^{c}}^{1,2}\left(\mathbb{R}_{+}^{N+1} ; t^{1-2 s}\right) .
$$

Hence we can replace $\phi=W$ in the previous identity thus obtaining that $\|W\|_{\mathcal{D}^{1,2}\left(\mathbb{R}_{+}^{N+1} ; t^{1-2 s}\right)}^{2}=0$ and hence $W \equiv 0$ in $\overline{\mathbb{R}_{+}^{N+1}}$, thus contradicting (4.2).

Lemma 4.2. Let $\left\{K_{\varepsilon}\right\}_{\varepsilon>0}$ be a family of compact sets contained in the open set $\Omega$ concentrating, in the sense of Definition [1.3, to a compact set $K \subset \Omega$, with $\operatorname{Cap}_{\Omega}^{s}(K)=0$. For every $u \in \mathcal{D}^{s, 2}(\Omega)$ it holds

$$
\lim _{\varepsilon \rightarrow 0^{+}} \operatorname{Cap}_{\Omega}^{s}\left(K_{\varepsilon}, u\right)=\operatorname{Cap}_{\Omega}^{s}(K, u)=0 \quad \text { and } \quad V_{\Omega, K_{\varepsilon}, u} \rightarrow V_{\Omega, K, u} \equiv 0
$$

strongly in $\mathcal{D}^{1,2}\left(\mathbb{R}_{+}^{N+1} ; t^{1-2 s}\right)$ as $\varepsilon \rightarrow 0^{+}$.

Proof. Let $U \in \mathcal{D}_{\Omega^{c}}^{1,2}\left(\mathbb{R}_{+}^{N+1} ; t^{1-2 s}\right)$ be such that $\operatorname{Tr} U=u$ and let $V_{\Omega, K_{\varepsilon}, u} \in \mathcal{D}_{\Omega^{c}}^{1,2}\left(\mathbb{R}_{+}^{N+1} ; t^{1-2 s}\right)$ achieve $\operatorname{Cap}_{\Omega}^{s}\left(K_{\varepsilon}, u\right)$. Then, by (2.23),$V_{\Omega, K_{\varepsilon}, u}-U \in \mathcal{D}_{\Omega^{c} \cup K_{\varepsilon}}^{1,2}\left(\mathbb{R}_{+}^{N+1} ; t^{1-2 s}\right)$ and

$$
q\left(V_{\Omega, K_{\varepsilon}, u}, \phi\right)=0 \quad \text { for all } \phi \in \mathcal{D}_{\Omega^{c} \cup K_{\varepsilon}}^{1,2}\left(\mathbb{R}_{+}^{N+1} ; t^{1-2 s}\right) .
$$

As $V_{\Omega, K_{\varepsilon}, u}$ achieves (2.9), we have

$$
\left\|V_{\Omega, K_{\varepsilon}, u}\right\|_{\mathcal{D}^{1,2}\left(\mathbb{R}_{+}^{N+1} ; t^{1-2 s}\right)}^{2} \leq\|U\|_{\mathcal{D}^{1,2}\left(\mathbb{R}_{+}^{N+1} ; t^{1-2 s}\right)}^{2}
$$

so that $\left\{V_{\Omega, K_{\varepsilon}, u}\right\}_{\varepsilon>0}$ is bounded in $\mathcal{D}_{\Omega^{c}}^{1,2}\left(\mathbb{R}_{+}^{N+1} ; t^{1-2 s}\right)$. Hence there exist a sequence $\varepsilon_{n} \rightarrow 0^{+}$and $V \in \mathcal{D}_{\Omega^{c}}^{1,2}\left(\mathbb{R}_{+}^{N+1} ; t^{1-2 s}\right)$ such that $V_{\Omega, K_{\varepsilon_{n}}, u} \rightarrow V$ weakly in $\mathcal{D}_{\Omega^{c}}^{1,2}\left(\mathbb{R}_{+}^{N+1} ; t^{1-2 s}\right)$. Let us show that $V=V_{\Omega, K, u}$. On the one hand, $V-U \in \mathcal{D}_{\Omega^{c} \cup K}^{1,2}\left(\mathbb{R}_{+}^{N+1} ; t^{1-2 s}\right)$ thanks to Proposition 2.6 and the assumption $\operatorname{Cap}_{\Omega}^{s}(K)=0$. On the other hand, passing to the limit in (4.3) we obtain that $q(V, \phi)=$ 0 for every $\phi \in C_{c}^{\infty}\left(\mathbb{R}_{+}^{N+1} \cup(\Omega \backslash K)\right)$ and so, by density, for every $\phi \in \mathcal{D}_{\Omega^{c} \cup K}^{1,2}\left(\mathbb{R}_{+}^{N+1} ; t^{1-2 s}\right)=$ $\mathcal{D}_{\Omega^{c}}^{1,2}\left(\mathbb{R}_{+}^{N+1} ; t^{1-2 s}\right)$. Therefore $V=V_{\Omega, K, u} \equiv 0$. In order to prove that the convergence is strong, take $\phi=V_{\Omega, K_{\varepsilon_{n}}, u}-U$ in (4.3) and pass to the limit to obtain

$$
\lim _{n \rightarrow+\infty} \operatorname{Cap}_{\Omega}^{s}\left(K_{\varepsilon_{n}}, u\right)=\lim _{n \rightarrow+\infty} q\left(V_{\Omega, K_{\varepsilon_{n}}, u}, V_{\Omega, K_{\varepsilon_{n}}, u}\right)=\lim _{n \rightarrow+\infty} q\left(V_{\Omega, K_{\varepsilon_{n}}, u}, U\right)=q(V, U)=0 .
$$

We conclude that $\operatorname{Cap}_{\Omega}^{s}\left(K_{\varepsilon_{n}}, u\right) \rightarrow 0$ and that $V_{\Omega, K_{\varepsilon_{n}}, u} \rightarrow 0$ strongly in $\mathcal{D}_{\Omega^{c}}^{1,2}\left(\mathbb{R}_{+}^{N+1} ; t^{1-2 s}\right)$. Since these limits do not depend on the sequence $\varepsilon_{n} \rightarrow 0$, we reach the conclusion.

Let us introduce the operator $A: \mathcal{D}_{\Omega^{c}}^{1,2}\left(\mathbb{R}_{+}^{N+1} ; t^{1-2 s}\right) \rightarrow \mathcal{D}_{\Omega^{c}}^{1,2}\left(\mathbb{R}_{+}^{N+1} ; t^{1-2 s}\right)$ defined by

$$
q(A(U), V)=\int_{\Omega} \operatorname{Tr} U \operatorname{TrV} d x
$$

for every $U, V \in \mathcal{D}_{\Omega^{c}}^{1,2}\left(\mathbb{R}_{+}^{N+1} ; t^{1-2 s}\right)$. It is straightforward to see that $A$ is symmetric, nonnegative, and compact. Letting, for $j \in \mathbb{N}_{*}$,

$$
\mu_{j}=\frac{1}{\kappa_{s} \lambda_{j}^{s}(\Omega)},
$$

the spectrum of $A$ is $\{0\} \cup\left\{\mu_{j}: j \in \mathbb{N}_{*}\right\}$; furthermore, since $\operatorname{dim} \operatorname{ker} A=+\infty, 0$ has infinite multiplicity as an eigenvalue of $A$, whereas the non-zero eigenvalues of $A$ have finite multiplicity. 
Proof of Theorem 1.5. Let $U_{j}=\mathcal{H}\left(u_{j}\right)$, so that $U_{j}$ satisfies (2.4) and (1.4). To simplify the notation, in the rest of the proof we write $V_{\varepsilon}=V_{\Omega, K_{\varepsilon}, u_{j}}$ and $\mathcal{H}_{\varepsilon}=\mathcal{D}_{\Omega^{c} \cup K_{\varepsilon}}^{1,2}\left(\mathbb{R}_{+}^{N+1} ; t^{1-2 s}\right)$. We divide the proof into three steps.

Step 1. We claim that

$$
\lambda_{j}^{s}\left(\Omega \backslash K_{\varepsilon}\right)-\lambda_{j}^{s}(\Omega)=o\left(\sqrt{\operatorname{Cap}_{\Omega}^{s}\left(K_{\varepsilon}, u_{j}\right)}\right) \quad \text { as } \varepsilon \rightarrow 0^{+} .
$$

Let

$$
\psi_{\varepsilon}=U_{j}-V_{\varepsilon} \in \mathcal{H}_{\varepsilon},
$$

so that $\psi_{\varepsilon}$ is the orthogonal projection of $U_{j}$ on $\mathcal{H}_{\varepsilon}$ in the space $\mathcal{D}_{\Omega^{c}}^{1,2}\left(\mathbb{R}_{+}^{N+1} ; t^{1-2 s}\right)$ endowed with the scalar product $q$, that is

$$
q\left(\psi_{\varepsilon}-U_{j}, \phi\right)=0 \quad \text { for every } \phi \in \mathcal{H}_{\varepsilon} .
$$

For every $\phi \in \mathcal{H}_{\varepsilon}$ we have, using (2.5),

$$
\begin{aligned}
q\left(\psi_{\varepsilon}, \phi\right)-\kappa_{s} \lambda_{j}^{s}(\Omega) \int_{\Omega} \operatorname{Tr} \psi_{\varepsilon} \operatorname{Tr} \phi d x & =q\left(U_{j}, \phi\right)-\kappa_{s} \lambda_{j}^{s}(\Omega) \int_{\Omega} \operatorname{Tr} \psi_{\varepsilon} \operatorname{Tr} \phi d x \\
& =\kappa_{s} \lambda_{j}^{s}(\Omega) \int_{\Omega} \operatorname{Tr} V_{\varepsilon} \operatorname{Tr} \phi d x
\end{aligned}
$$

so that

$$
\int_{\Omega} \operatorname{Tr} \psi_{\varepsilon} \operatorname{Tr} \phi d x=\frac{1}{\kappa_{s} \lambda_{j}^{s}(\Omega)} q\left(\psi_{\varepsilon}, \phi\right)-\int_{\Omega} \operatorname{Tr} V_{\varepsilon} \operatorname{Tr} \phi d x \quad \text { for ever } \phi \in \mathcal{H}_{\varepsilon} .
$$

Let $A_{\varepsilon}: \mathcal{H}_{\varepsilon} \rightarrow \mathcal{H}_{\varepsilon}$ be defined by

$$
q\left(A_{\varepsilon}(U), V\right)=\int_{\Omega} \operatorname{Tr} U \operatorname{TrV} d x \quad \text { for every } U, V \in \mathcal{H}_{\varepsilon} .
$$

Recalling the definition of $\mu_{j}$ in (4.5), the spectral theorem (see for instance [21, Proposition 8.20]) provides

$$
\operatorname{dist}\left(\mu_{j}, \sigma\left(A_{\varepsilon}\right)\right) \leq \frac{\left\|A_{\varepsilon} \psi_{\varepsilon}-\mu_{j} \psi_{\varepsilon}\right\|_{\mathcal{H}_{\varepsilon}}}{\left\|\psi_{\varepsilon}\right\|_{\mathcal{H}_{\varepsilon}}}
$$

where $\sigma\left(A_{\varepsilon}\right)$ is the spectrum of $A_{\varepsilon}$.

Taking into account Lemma 4.2, we have that

$$
\left|q\left(U_{j}, V_{\varepsilon}\right)\right| \leq \sqrt{q\left(U_{j}, U_{j}\right)} \sqrt{q\left(V_{\varepsilon}, V_{\varepsilon}\right)}=\sqrt{\lambda_{j}^{s}(\Omega) \kappa_{s}} \sqrt{\operatorname{Cap}_{\Omega}^{s}\left(K_{\varepsilon}, u_{j}\right)}=o(1)
$$

as $\varepsilon \rightarrow 0$, then the denominator in the right hand side of (4.10) is easily estimated as follows

$$
\begin{aligned}
\left\|\psi_{\varepsilon}\right\|_{\mathcal{H}_{\varepsilon}}^{2} & =q\left(U_{j}-V_{\varepsilon}, U_{j}-V_{\varepsilon}\right)=q\left(U_{j}, U_{j}\right)+\operatorname{Cap}_{\Omega}^{s}\left(K_{\varepsilon}, u_{j}\right)-2 q\left(U_{j}, V_{\varepsilon}\right) \\
& =\lambda_{j}^{s}(\Omega) \kappa_{s}+o(1) \quad \text { as } \varepsilon \rightarrow 0^{+} .
\end{aligned}
$$

In order to estimate the numerator in the right hand side of (4.10), let $Z_{\varepsilon}=A_{\varepsilon} \psi_{\varepsilon}-\mu_{j} \psi_{\varepsilon} \in \mathcal{H}_{\varepsilon}$. Using (4.9) and (4.8), we have

$$
q\left(Z_{\varepsilon}, \phi\right)+\mu_{j} q\left(\psi_{\varepsilon}, \phi\right)=q\left(A_{\varepsilon} \psi_{\varepsilon}, \phi\right)=\int_{\Omega} \operatorname{Tr} \psi_{\varepsilon} \operatorname{Tr} \phi d x=\mu_{j} q\left(\psi_{\varepsilon}, \phi\right)-\int_{\Omega} \operatorname{Tr} V_{\varepsilon} \operatorname{Tr} \phi d x,
$$

for every $\phi \in \mathcal{H}_{\varepsilon}$. Choosing $\phi=Z_{\varepsilon} \in \mathcal{H}_{\varepsilon}$ in the previous expression and using Theorem 2.3 (i) and (2.3), we obtain

$$
\begin{aligned}
\left\|Z_{\varepsilon}\right\|_{\mathcal{H}_{\varepsilon}}^{2} & =-\int_{\Omega} \operatorname{Tr} V_{\varepsilon} \operatorname{Tr} Z_{\varepsilon} d x \leq\left\|\operatorname{Tr} V_{\varepsilon}\right\|_{L^{2}(\Omega)}|\Omega|^{\frac{s}{N}}\left(\int_{\Omega}\left|\operatorname{Tr} Z_{\varepsilon}\right|^{2^{*}(s)}\right)^{\frac{1}{2^{*}(s)}} \\
& \leq\left\|\operatorname{Tr} V_{\varepsilon}\right\|_{L^{2}(\Omega)}|\Omega|^{\frac{s}{N}} S_{N, s}^{-1}\left\|\operatorname{Tr} Z_{\varepsilon}\right\|_{\mathcal{D}^{s, 2}\left(\mathbb{R}^{N}\right)} \leq\left\|\operatorname{Tr} V_{\varepsilon}\right\|_{L^{2}(\Omega)}|\Omega|^{\frac{s}{N}} S_{N, s}^{-1} \kappa_{s}^{-1 / 2}\left\|Z_{\varepsilon}\right\|_{\mathcal{H}_{\varepsilon}} .
\end{aligned}
$$

Replacing (4.11) and (4.12) into (4.10), we find that there exists a constant $C$ independent of $\varepsilon$ such that

$$
\operatorname{dist}\left(\mu_{j}, \sigma\left(A_{\varepsilon}\right)\right) \leq C\left\|\operatorname{Tr} V_{\varepsilon}\right\|_{L^{2}(\Omega)} .
$$


Now, the assumption that $\lambda_{j}^{s}(\Omega)$ is simple and the continuity proved in Theorem 1.2 imply that $\lambda_{j, \varepsilon}:=\lambda_{j}^{s}\left(\Omega \backslash K_{\varepsilon}\right) \quad$ is simple for $\varepsilon>0$ small enough.

Denoting as

$$
\mu_{j, \varepsilon}=1 /\left(\kappa_{s} \lambda_{j, \varepsilon}\right)
$$

the $j$-th eigenvalue of $A_{\varepsilon}$, by the simplicity of $\mu_{j}$ as an eigenvalue of the operator $A$ introduced in (4.4), and by Theorem 1.2 we have that

$$
\operatorname{dist}\left(\mu_{j}, \sigma\left(A_{\varepsilon}\right)\right)=\left|\mu_{j}-\mu_{j, \varepsilon}\right| \quad \text { for } \varepsilon>0 \text { small enough. }
$$

Then relation (4.13) provides, for $\varepsilon$ small enough,

$$
\left|\lambda_{j}^{s}(\Omega)-\lambda_{j, \varepsilon}\right|=\kappa_{s} \lambda_{j}^{s}(\Omega) \lambda_{j, \varepsilon}\left|\mu_{j}-\mu_{j, \varepsilon}\right| \leq C \kappa_{s} \lambda_{j}^{s}(\Omega) \lambda_{j, \varepsilon}\left\|\operatorname{Tr} V_{\varepsilon}\right\|_{L^{2}(\Omega)} .
$$

As $C$ is independent of $\varepsilon$ and $\lim _{\varepsilon \rightarrow 0^{+}} \lambda_{j, \varepsilon}=\lambda_{j}^{s}(\Omega)$, Lemma 4.1 provides the claim.

Step 2. We claim that

$$
\left\|\psi_{\varepsilon}-\Pi_{\varepsilon} \psi_{\varepsilon}\right\|_{\mathcal{H}_{\varepsilon}}=o\left(\sqrt{\operatorname{Cap}_{\Omega}^{s}\left(K_{\varepsilon}, u_{j}\right)}\right) \quad \text { as } \varepsilon \rightarrow 0^{+},
$$

where $\Pi_{\varepsilon}: \mathcal{D}_{\Omega^{c}}^{1,2}\left(\mathbb{R}_{+}^{N+1} ; t^{1-2 s}\right) \rightarrow \mathcal{H}_{\varepsilon}$ is defined as

$$
\Pi_{\varepsilon} W=\left(\int_{\Omega} \operatorname{Tr} W \operatorname{Tr} U_{j, \varepsilon} d x\right) U_{j, \varepsilon} \quad \text { for any } W \in \mathcal{D}_{\Omega^{c}}^{1,2}\left(\mathbb{R}_{+}^{N+1} ; t^{1-2 s}\right)
$$

and $U_{j, \varepsilon}$ is a normalized eigenfunction associated to $\lambda_{j, \varepsilon}$, i.e.

$$
\left\{\begin{array}{l}
U_{j, \varepsilon} \in \mathcal{H}_{\varepsilon}, \\
q\left(U_{j, \varepsilon}, \phi\right)=\lambda_{j, \varepsilon} \kappa_{s} \int_{\Omega} \operatorname{Tr} U_{j, \varepsilon} \operatorname{Tr} \phi d x \quad \text { for every } \phi \in \mathcal{H}_{\varepsilon}, \\
\int_{\Omega}\left|\operatorname{Tr} U_{j, \varepsilon}(x)\right|^{2} d x=1 .
\end{array}\right.
$$

Let $\tilde{U}_{\varepsilon}=\psi_{\varepsilon}-\Pi_{\varepsilon} \psi_{\varepsilon}$ and notice that

$$
\int_{\Omega} \operatorname{Tr} \tilde{U}_{\varepsilon} \operatorname{Tr} U_{j, \varepsilon} d x=0 .
$$

Using the fact that $\Pi_{\varepsilon} \psi_{\varepsilon}$ is an eigenfunction associated to $\lambda_{j, \varepsilon}$ and relation (4.8), we see that the following holds for every $\phi \in \mathcal{H}_{\varepsilon}$

$$
\begin{aligned}
& q\left(\tilde{U}_{\varepsilon}, \phi\right)-\kappa_{s} \lambda_{j, \varepsilon} \int_{\Omega} \operatorname{Tr} \tilde{U}_{\varepsilon} \operatorname{Tr} \phi d x \\
& \quad=q\left(\psi_{\varepsilon}, \phi\right)-\kappa_{s} \lambda_{j, \varepsilon} \int_{\Omega} \operatorname{Tr} \psi_{\varepsilon} \operatorname{Tr} \phi d x-\left[q\left(\Pi_{\varepsilon} \psi_{\varepsilon}, \phi\right)-\kappa_{s} \lambda_{j, \varepsilon} \int_{\Omega} \operatorname{Tr}\left(\Pi_{\varepsilon} \psi_{\varepsilon}\right) \operatorname{Tr} \phi d x\right] \\
& \quad=q\left(\psi_{\varepsilon}, \phi\right)-\kappa_{s} \lambda_{j}^{s}(\Omega) \int_{\Omega} \operatorname{Tr} \psi_{\varepsilon} \operatorname{Tr} \phi d x+\kappa_{s}\left(\lambda_{j}^{s}(\Omega)-\lambda_{j, \varepsilon}\right) \int_{\Omega} \operatorname{Tr} \psi_{\varepsilon} \operatorname{Tr} \phi d x \\
& \quad=\kappa_{s} \lambda_{j}^{s}(\Omega) \int_{\Omega} \operatorname{Tr} V_{\varepsilon} \operatorname{Tr} \phi d x+\kappa_{s}\left(\lambda_{j}^{s}(\Omega)-\lambda_{j, \varepsilon}\right) \int_{\Omega} \operatorname{Tr} \psi_{\varepsilon} \operatorname{Tr} \phi d x .
\end{aligned}
$$

Let $\xi_{\varepsilon}=A_{\varepsilon}\left(\tilde{U}_{\varepsilon}\right)-\mu_{j, \varepsilon} \tilde{U}_{\varepsilon} \in \mathcal{H}_{\varepsilon}$. We use the definition of $A_{\varepsilon}$ in (4.9), that of $\mu_{j, \varepsilon}$ in (4.14) and relation (4.18) evaluated at $\phi=\xi_{\varepsilon}$ to compute

$$
\begin{aligned}
\left\|\xi_{\varepsilon}\right\|_{\mathcal{H}_{\varepsilon}}^{2} & =q\left(A_{\varepsilon}\left(\tilde{U}_{\varepsilon}\right), \xi_{\varepsilon}\right)-\mu_{j, \varepsilon} q\left(\tilde{U}_{\varepsilon}, \xi_{\varepsilon}\right)=\int_{\Omega} \operatorname{Tr} \tilde{U}_{\varepsilon} \operatorname{Tr} \xi_{\varepsilon} d x \\
- & {\left[\int_{\Omega} \operatorname{Tr} \tilde{U}_{\varepsilon} \operatorname{Tr} \xi_{\varepsilon} d x+\frac{\lambda_{j}^{s}(\Omega)}{\lambda_{j, \varepsilon}} \int_{\Omega} \operatorname{Tr} V_{\varepsilon} \operatorname{Tr} \xi_{\varepsilon} d x+\frac{\lambda_{j}^{s}(\Omega)-\lambda_{j, \varepsilon}}{\lambda_{j, \varepsilon}} \int_{\Omega} \operatorname{Tr} \psi_{\varepsilon} \operatorname{Tr} \xi_{\varepsilon} d x\right] } \\
& =-\frac{\lambda_{j}^{s}(\Omega)}{\lambda_{j, \varepsilon}} \int_{\Omega} \operatorname{Tr} V_{\varepsilon} \operatorname{Tr} \xi_{\varepsilon} d x-\frac{\lambda_{j}^{s}(\Omega)-\lambda_{j, \varepsilon}}{\lambda_{j, \varepsilon}} \int_{\Omega} \operatorname{Tr} \psi_{\varepsilon} \operatorname{Tr} \xi_{\varepsilon} d x
\end{aligned}
$$

from which, taking into account (4.11) and (2.14), we deduce that

$$
\left\|\xi_{\varepsilon}\right\|_{\mathcal{H}_{\varepsilon}} \leq C\left(\left\|\operatorname{Tr} V_{\varepsilon}\right\|_{L^{2}(\Omega)}+\left|\lambda_{j}^{s}(\Omega)-\lambda_{j, \varepsilon}\right|\right)
$$


for a constant $C$ not depending on $\varepsilon$. Lemma 4.1 and relation (4.6) provide then

$$
\left\|A_{\varepsilon}\left(\tilde{U}_{\varepsilon}\right)-\mu_{j, \varepsilon} \tilde{U}_{\varepsilon}\right\|_{\mathcal{H}_{\varepsilon}}=\left\|\xi_{\varepsilon}\right\|_{\mathcal{H}_{\varepsilon}}=o\left(\sqrt{\operatorname{Cap}_{\Omega}^{s}\left(K_{\varepsilon}, u_{j}\right)}\right) \quad \text { as } \varepsilon \rightarrow 0^{+} .
$$

Let

$$
\mathcal{K}_{\varepsilon}=\left.\operatorname{Ker} \Pi_{\varepsilon}\right|_{\mathcal{H}_{\varepsilon}}=\left\{W \in \mathcal{H}_{\varepsilon}: \int_{\Omega} \operatorname{Tr} W \operatorname{Tr} U_{j, \varepsilon} d x=0\right\}
$$

and note that $\tilde{U}_{\varepsilon} \in \mathcal{K}_{\varepsilon}$ thanks to (4.17). Moreover, in view of (4.9) and (4.16), $A_{\varepsilon}(U) \in \mathcal{K}_{\varepsilon}$ for all $U \in \mathcal{K}_{\varepsilon}$, hence, denoting as $\tilde{A}_{\varepsilon}$ the restriction of $A_{\varepsilon}$ to $\mathcal{K}_{\varepsilon}$, we have $\tilde{A}_{\varepsilon}: \mathcal{K}_{\varepsilon} \rightarrow \mathcal{K}_{\varepsilon}$. As $\sigma\left(\tilde{A}_{\varepsilon}\right)=\sigma\left(A_{\varepsilon}\right) \backslash\left\{\mu_{j, \varepsilon}\right\}$, there exists $\delta>0$ independent of $\varepsilon$ such that $\operatorname{dist}\left(\mu_{j, \varepsilon}, \sigma\left(\tilde{A}_{\varepsilon}\right)\right) \geq \delta$. We use this inequality, the spectral theorem, and relation (4.19) to obtain

$$
\begin{aligned}
\left\|\psi_{\varepsilon}-\Pi_{\varepsilon} \psi_{\varepsilon}\right\|_{\mathcal{H}_{\varepsilon}}=\left\|\tilde{U}_{\varepsilon}\right\|_{\mathcal{H}_{\varepsilon}} & \leq \frac{1}{\delta} \operatorname{dist}\left(\mu_{j, \varepsilon}, \sigma\left(\tilde{A}_{\varepsilon}\right)\right)\left\|\tilde{U}_{\varepsilon}\right\|_{\mathcal{H}_{\varepsilon}} \\
& \leq \frac{1}{\delta}\left\|\tilde{A}_{\varepsilon}\left(\tilde{U}_{\varepsilon}\right)-\mu_{j, \varepsilon} \tilde{U}_{\varepsilon}\right\|_{\mathcal{H}_{\varepsilon}}=o\left(\sqrt{\operatorname{Cap}_{\Omega}^{s}\left(K_{\varepsilon}, u_{j}\right)}\right)
\end{aligned}
$$

as $\varepsilon \rightarrow 0^{+}$, thus proving (4.15).

Step 3. From the definition of $\psi_{\varepsilon}$ (4.7), (1.4), Lemma 4.1, (4.15) and (2.14), we have

$$
\begin{aligned}
\left\|\operatorname{Tr}\left(\Pi_{\varepsilon} \psi_{\varepsilon}\right)\right\|_{L^{2}(\Omega)} & =\left(\int_{\Omega}\left|\operatorname{Tr}\left(\Pi_{\varepsilon} \psi_{\varepsilon}-\psi_{\varepsilon}\right)+u_{j}-\operatorname{Tr} V_{\varepsilon}\right|^{2} d x\right)^{1 / 2} \\
& =\left(1+o\left(\sqrt{\operatorname{Cap}_{\Omega}^{s}\left(K_{\varepsilon}, u_{j}\right)}\right)\right)^{1 / 2} \\
& =1+o\left(\sqrt{\operatorname{Cap}_{\Omega}^{s}\left(K_{\varepsilon}, u_{j}\right)}\right) \quad \text { as } \varepsilon \rightarrow 0^{+} .
\end{aligned}
$$

Let

Noticing that

$$
\Psi_{\varepsilon}=\frac{\Pi_{\varepsilon} \psi_{\varepsilon}}{\left\|\operatorname{Tr}\left(\Pi_{\varepsilon} \psi_{\varepsilon}\right)\right\|_{L^{2}(\Omega)}} \in \mathcal{H}_{\varepsilon}
$$

$$
\Psi_{\varepsilon}-\psi_{\varepsilon}=\frac{\Pi_{\varepsilon} \psi_{\varepsilon}-\psi_{\varepsilon}+\left(1-\left\|\operatorname{Tr}\left(\Pi_{\varepsilon} \psi_{\varepsilon}\right)\right\|_{L^{2}(\Omega)}\right) \psi_{\varepsilon}}{\left\|\operatorname{Tr}\left(\Pi_{\varepsilon} \psi_{\varepsilon}\right)\right\|_{L^{2}(\Omega)}}
$$

and using (4.20), (4.15) and (2.14), we deduce that

$$
\left\|\operatorname{Tr}\left(\Psi_{\varepsilon}-\psi_{\varepsilon}\right)\right\|_{L^{2}(\Omega)}=o\left(\sqrt{\operatorname{Cap}_{\Omega}^{s}\left(K_{\varepsilon}, u_{j}\right)}\right) \quad \text { as } \varepsilon \rightarrow 0^{+} .
$$

Similarly,

$$
\left\|\operatorname{Tr}\left(\Psi_{\varepsilon}-U_{j}\right)\right\|_{L^{2}(\Omega)}=\left\|\operatorname{Tr}\left(\Psi_{\varepsilon}-\psi_{\varepsilon}-V_{\varepsilon}\right)\right\|_{L^{2}(\Omega)}=o\left(\sqrt{\operatorname{Cap}_{\Omega}^{s}\left(K_{\varepsilon}, u_{j}\right)}\right) \quad \text { as } \varepsilon \rightarrow 0^{+} .
$$

We also remark, using the equation satisfied by $V_{\varepsilon}$ (see (2.23) $)$, the fact that $\psi_{\varepsilon} \in \mathcal{H}_{\varepsilon}$ and the equation satisfied by $U_{j}$, that

$$
\operatorname{Cap}_{\Omega}^{s}\left(K_{\varepsilon}, u_{j}\right)=\frac{1}{\kappa_{s}} q\left(V_{\varepsilon}, V_{\varepsilon}\right)=\frac{1}{\kappa_{s}} q\left(V_{\varepsilon}, U_{j}-\psi_{\varepsilon}\right)=\frac{1}{\kappa_{s}} q\left(V_{\varepsilon}, U_{j}\right)=\lambda_{j}^{s}(\Omega) \int_{\Omega} u_{j} \operatorname{Tr} V_{\varepsilon} d x .
$$

Noticing that $\Psi_{\varepsilon}$ is an eigenfunction associated to $\lambda_{j, \varepsilon}$, relation (4.8) with $\phi=\Psi_{\varepsilon}$ provides

$$
\left(\lambda_{j, \varepsilon}-\lambda_{j}^{s}(\Omega)\right) \int_{\Omega} \operatorname{Tr} \Psi_{\varepsilon} \operatorname{Tr} \psi_{\varepsilon} d x=\lambda_{j}^{s}(\Omega) \int_{\Omega} \operatorname{Tr} \Psi_{\varepsilon} \operatorname{Tr} V_{\varepsilon} d x .
$$

Therefore, by (4.22), (4.23) and Lemma 4.1, we have

$$
\begin{aligned}
\left(\lambda_{j, \varepsilon}-\lambda_{j}^{s}(\Omega)\right) \int_{\Omega} \operatorname{Tr} \Psi_{\varepsilon} \operatorname{Tr} \psi_{\varepsilon} d x & =\lambda_{j}^{s}(\Omega) \int_{\Omega} u_{j} \operatorname{Tr} V_{\varepsilon} d x+\lambda_{j}^{s}(\Omega) \int_{\Omega} \operatorname{Tr}\left(\Psi_{\varepsilon}-U_{j}\right) \operatorname{Tr} V_{\varepsilon} d x \\
& =\operatorname{Cap}_{\Omega}^{s}\left(K_{\varepsilon}, u_{j}\right)+o\left(\operatorname{Cap}_{\Omega}^{s}\left(K_{\varepsilon}, u_{j}\right)\right)
\end{aligned}
$$


as $\varepsilon \rightarrow 0^{+}$. As, by (4.21),

$$
\int_{\Omega} \operatorname{Tr} \Psi_{\varepsilon} \operatorname{Tr} \psi_{\varepsilon} d x=\int_{\Omega}\left|\operatorname{Tr} \Psi_{\varepsilon}\right|^{2} d x+\int_{\Omega} \operatorname{Tr} \Psi_{\varepsilon} \operatorname{Tr}\left(\psi_{\varepsilon}-\Psi_{\varepsilon}\right) d x=1+o\left(\sqrt{\operatorname{Cap}_{\Omega}^{s}\left(K_{\varepsilon}, u_{j}\right)}\right),
$$

we have concluded the proof.

\section{Asymptotics of CAPacities For SCALING OF A Given SET}

In this section we will assume that $0 \in \Omega$. In order to prove Theorem 1.6 we first establish the following preliminary result.

Lemma 5.1. Let $K \subset \Omega$ be compact and $\Omega^{\prime}$ be an open set such that $K \subset \Omega^{\prime} \Subset \Omega$. Let $f \in H_{\mathrm{loc}}^{s}(\Omega)$ and $\left(f_{n}\right)_{n \geq 1} \subset H_{\mathrm{loc}}^{s}(\Omega)$ be such that $f_{n} \rightarrow f$ as $n \rightarrow+\infty$ in $H^{s}\left(\Omega^{\prime}\right)$. Then

$$
V_{\Omega, K, f_{n}} \rightarrow V_{\Omega, K, f} \quad \text { in } \mathcal{D}^{1,2}\left(\mathbb{R}^{N+1} ; t^{1-2 s}\right)
$$

and

$$
\lim _{n \rightarrow+\infty} \operatorname{Cap}_{\Omega}^{s}\left(K, f_{n}\right)=\operatorname{Cap}_{\Omega}^{s}(K, f) .
$$

Proof. Let $\tilde{\eta}_{K} \in C^{\infty}\left(\mathbb{R}_{+}^{N+1} \cup \Omega^{\prime}\right)$ be such that $\tilde{\eta}_{K} \equiv 1$ in a neighborhood of $K$. Therefore $\tilde{\eta}_{K} f_{n} \rightarrow \tilde{\eta}_{K} f$ in $\mathcal{D}^{s, 2}\left(\Omega^{\prime}\right)$ and, consequently, $\mathcal{H}\left(\tilde{\eta}_{K} f_{n}\right) \rightarrow \mathcal{H}\left(\tilde{\eta}_{K} f\right)$ in $\mathcal{D}^{1,2}\left(\mathbb{R}^{N+1} ; t^{1-2 s}\right)$, where $\mathcal{H}$ is the extension operator introduced in (2.2). Hence

Furthermore both $V_{\Omega, K, f_{n}}-\mathcal{H}\left(\tilde{\eta}_{K} f_{n}\right)$ and $V_{\Omega, K, f}-\mathcal{H}\left(\tilde{\eta}_{K} f\right)$ belong to $\mathcal{D}_{\Omega^{c} \cup K}^{1,2}\left(\mathbb{R}^{N+1} ; t^{1-2 s}\right)$.

$$
q\left(V_{\Omega, K, f_{n}}-V_{\Omega, K, f}, V_{\Omega, K, f_{n}}-\mathcal{H}\left(\tilde{\eta}_{K} f_{n}\right)\right)=q\left(V_{\Omega, K, f_{n}}-V_{\Omega, K, f}, V_{\Omega, K, f}-\mathcal{H}\left(\tilde{\eta}_{K} f\right)\right)=0,
$$

so that, using the Hölder inequality,

$$
\begin{aligned}
\left\|V_{\Omega, K, f_{n}}-V_{\Omega, K, f}\right\|_{\mathcal{D}^{1,2}\left(\mathbb{R}^{N+1} ; t^{1-2 s}\right)}^{2}=q\left(V_{\Omega, K, f_{n}}-V_{\Omega, K, f}, \mathcal{H}\left(\tilde{\eta}_{K} f_{n}\right)-\mathcal{H}\left(\tilde{\eta}_{K} f\right)\right) \\
\leq\left\|V_{\Omega, K, f_{n}}-V_{\Omega, K, f}\right\|_{\mathcal{D}^{1,2}\left(\mathbb{R}^{N+1} ; t^{1-2 s}\right)}\left\|\mathcal{H}\left(\tilde{\eta}_{K} f_{n}\right)-\mathcal{H}\left(\tilde{\eta}_{K} f\right)\right\|_{\mathcal{D}^{1,2}\left(\mathbb{R}^{N+1} ; t^{1-2 s}\right)}
\end{aligned}
$$

Then

concluding the proof.

$$
\lim _{n \rightarrow+\infty}\left\|V_{\Omega, K, f_{n}}-V_{\Omega, K, f}\right\|_{\mathcal{D}^{1,2}\left(\mathbb{R}^{N+1} ; t^{1-2 s}\right)}=0
$$

Proof of Theorem [1.6. For every $\varepsilon>0$, let $V_{\Omega, K_{\varepsilon}, u_{j}}$ be the function that achieves $\operatorname{Cap}_{\Omega}^{s}\left(K_{\varepsilon}, u_{j}\right)$ as in (2.21) and let

$$
\tilde{V}_{\varepsilon}(z)=\varepsilon^{-\gamma_{s}} V_{\Omega, K_{\varepsilon}, u_{j}}(\varepsilon z), \quad z \in \mathbb{R}_{+}^{N+1} .
$$

Let $U_{j}=\mathcal{H}\left(u_{j}\right) \in \mathcal{D}_{\Omega^{c}}^{1,2}\left(\mathbb{R}_{+}^{N+1} ; t^{1-2 s}\right)$ be the extension of $u_{j}$ as in (2.2) and define $\tilde{U}_{\varepsilon}(z):=$ $\varepsilon^{-\gamma_{s}} U_{j}(\varepsilon z)$ as in Section 2.2

We notice that $\tilde{V}_{\varepsilon} \in \mathcal{D}_{(\Omega / \varepsilon)^{c}}^{1,2}\left(\mathbb{R}_{+}^{N+1} ; t^{1-2 s}\right), \tilde{V}_{\varepsilon}-\tilde{U}_{\varepsilon} \in \mathcal{D}_{(\Omega / \varepsilon)^{c} \cup K}^{1,2}\left(\mathbb{R}_{+}^{N+1} ; t^{1-2 s}\right)$ and

$$
q\left(\tilde{V}_{\varepsilon}, \phi\right)=0 \quad \text { for all } \phi \in \mathcal{D}_{(\Omega / \varepsilon)^{c} \cup K}^{1,2}\left(\mathbb{R}_{+}^{N+1} ; t^{1-2 s}\right) .
$$

In particular,

$$
\left\|\tilde{V}_{\varepsilon}\right\|_{\mathcal{D}^{1,2}\left(\mathbb{R}^{N+1} ; t^{1-2 s}\right)}^{2}=\kappa_{s} \operatorname{Cap}_{\Omega / \varepsilon}^{s}\left(K, \tilde{u}_{\varepsilon}\right),
$$

where $\tilde{u}_{\varepsilon}=\operatorname{Tr} \tilde{U}_{\varepsilon}$.

Let $r_{0}>0$ be such that $K \subset B_{r_{0}}^{\prime}=\left\{x \in \mathbb{R}^{N}:|x|<r_{0}\right\}$. For $\varepsilon$ sufficiently small, we have that

$$
B_{r_{0}}^{\prime} \subset \frac{\Omega}{\varepsilon}
$$

so that $\mathcal{D}_{\left(B_{r_{0}}^{\prime}\right)}^{1,2} \cup K \subseteq \mathcal{D}_{(\Omega / \varepsilon)^{c} \cup K}^{1,2}$ and, in turn,

$$
\operatorname{Cap}_{\Omega / \varepsilon}^{s}\left(K, \tilde{u}_{\varepsilon}\right) \leq \operatorname{Cap}_{B_{r_{0}}^{\prime}}^{s}\left(K, \tilde{u}_{\varepsilon}\right) \rightarrow \operatorname{Cap}_{B_{r_{0}}^{\prime}}^{s}(K, \hat{\psi})
$$


as $\varepsilon \rightarrow 0^{+}$, where in the last step we used (1.9) and Lemma 5.1. Combining (5.2) and (5.3), we deduce that the family $\left\{\tilde{V}_{\varepsilon}\right\}_{\varepsilon>0}$ is bounded in the reflexive space $\mathcal{D}^{1,2}\left(\mathbb{R}_{+}^{N+1} ; t^{1-2 s}\right)$. Then there exist a sequence $\varepsilon_{n} \rightarrow 0^{+}$and $\tilde{V} \in \mathcal{D}^{1,2}\left(\mathbb{R}_{+}^{N+1} ; t^{1-2 s}\right)$ such that

$$
\tilde{V}_{\varepsilon_{n}} \rightarrow \tilde{V} \quad \text { weakly in } \mathcal{D}^{1,2}\left(\mathbb{R}_{+}^{N+1} ; t^{1-2 s}\right)
$$

as $n \rightarrow+\infty$.

Let $\tilde{\eta}_{K} \in C_{c}^{\infty}\left(B_{R}^{+}\right)$for some $R>0$, be such that $\tilde{\eta}_{k}=1$ on a neighborhood of $K$. Then $\tilde{V}_{\varepsilon_{n}}-\tilde{\eta}_{K} \tilde{U}_{\varepsilon_{n}} \in \mathcal{D}_{K}^{1,2}\left(\mathbb{R}_{+}^{N+1} ; t^{1-2 s}\right)=\left\{U \in \mathcal{D}^{1,2}\left(\mathbb{R}_{+}^{N+1} ; t^{1-2 s}\right): \operatorname{Tr} U \in \mathcal{D}^{s, 2}\left(\mathbb{R}^{N} \backslash K\right)\right\}$. Moreover, by (2.10) we have that

$$
\tilde{\eta}_{K} \tilde{U}_{\varepsilon} \rightarrow \tilde{\eta}_{K} \tilde{\psi} \quad \text { in } \mathcal{D}^{1,2}\left(\mathbb{R}_{+}^{N+1} ; t^{1-2 s}\right) .
$$

Since $\mathcal{D}_{K}^{1,2}\left(\mathbb{R}_{+}^{N+1} ; t^{1-2 s}\right)$ is closed in $\mathcal{D}^{1,2}\left(\mathbb{R}_{+}^{N+1} ; t^{1-2 s}\right)$ (in the strong topology and then, being a subspace, in the weak topology), by (5.4) we conclude that $\tilde{V}-\tilde{\eta}_{k} \tilde{\psi} \in \mathcal{D}_{K}^{1,2}\left(\mathbb{R}_{+}^{N+1} ; t^{1-2 s}\right)$. Moreover, relations (5.1) and (5.4) provide

$$
q(\tilde{V}, \phi)=0 \quad \text { for all } \phi \in C_{c}^{\infty}\left(\mathbb{R}_{+}^{N+1} \cup\left(\mathbb{R}^{N} \backslash K\right)\right),
$$

so that, by density,

$$
q(\tilde{V}, \phi)=0 \quad \text { for all } \phi \in \mathcal{D}_{K}^{1,2}\left(\mathbb{R}_{+}^{N+1} ; t^{1-2 s}\right) .
$$

In particular,

$$
\|\tilde{V}\|_{\mathcal{D}^{1,2}\left(\mathbb{R}_{+}^{N+1} ; t^{1-2 s}\right)}^{2}=\kappa_{s} \operatorname{Cap}_{\mathbb{R}^{N}}^{s}(K, \hat{\psi})=q\left(\tilde{V}, \tilde{\eta}_{K} \tilde{\psi}\right) .
$$

Similarly, since $\tilde{V}_{\varepsilon}-\tilde{\eta}_{K} \tilde{U}_{\varepsilon} \in \mathcal{D}_{(\Omega / \varepsilon)^{c} \cup K}^{1,2}\left(\mathbb{R}_{+}^{N+1} ; t^{1-2 s}\right)$ for $\varepsilon>0$ sufficiently small, using also relations (5.1), (5.4), (5.5) and (5.6), we obtain

$$
\left\|\tilde{V}_{\varepsilon_{n}}\right\|_{\mathcal{D}^{1,2}\left(\mathbb{R}_{+}^{N+1} ; t^{1-2 s}\right)}^{2}=q\left(\tilde{V}_{\varepsilon_{n}}, \tilde{\eta}_{K} \tilde{U}_{\varepsilon_{n}}\right) \rightarrow q\left(\tilde{V}, \tilde{\eta}_{K} \tilde{\psi}\right)=\kappa_{s} \operatorname{Cap}_{\mathbb{R}^{N}}^{s}(K, \hat{\psi}),
$$

as $n \rightarrow+\infty$. By the Urysohn's subsequence principle we conclude that the above convergence holds as $\varepsilon \rightarrow 0^{+}$and not only along the sequence $\varepsilon_{n}$. To conclude the proof it suffices to notice that, by a change of variables,

$$
\operatorname{Cap}_{\Omega}^{s}\left(K_{\varepsilon}, u_{j}\right)=\frac{1}{\kappa_{s}}\left\|V_{\Omega, K_{\varepsilon}, u_{j}}\right\|_{\mathcal{D}^{1,2}\left(\mathbb{R}_{+}^{N+1} ; t^{1-2 s}\right)}^{2}=\frac{1}{\kappa_{s}} \varepsilon^{N+2\left(\gamma_{s}-s\right)}\left\|\tilde{V}_{\varepsilon}\right\|_{\mathcal{D}^{1,2}\left(\mathbb{R}_{+}^{N+1} ; t^{1-2 s}\right)}^{2}
$$

and to replace (5.7) into the previous expression.

Proof of Theorem 1.7. The family of sets $\{\varepsilon K\}_{\varepsilon>0}$ concentrates to the compact set $\{0\}$, which satisfies $\operatorname{Cap}_{\Omega}^{s}(\{0\})=0$ by Example 2.5, so that Theorem 1.5 applies in our situation. By combining it with Theorem 1.6, we obtain the stated result.

Proof of Corollary 1.8. Let $V_{K}$ be the function that achieves the infimum in (2.9) with $u=\hat{\psi}$ and $\Omega=\mathbb{R}^{N}$, so that $\operatorname{Cap}_{\mathbb{R}^{N}}^{s}(K, \hat{\psi})=\frac{1}{\kappa_{s}} q\left(V_{K}, V_{K}\right)$. The Hardy-trace inequality (2.13) provides

$$
\begin{aligned}
\operatorname{Cap}_{\mathbb{R}^{N}}^{s}(K, \hat{\psi})=\frac{1}{\kappa_{s}} q\left(V_{K}, V_{K}\right) & \geq \Lambda_{N, s} \int_{\mathbb{R}^{N}} \frac{\left|\operatorname{Tr} V_{K}\right|^{2}}{|x|^{2 s}} d x \\
& \geq \Lambda_{N, s} \int_{K} \frac{\left|\operatorname{Tr} V_{K}\right|^{2}}{|x|^{2 s}} d x=\Lambda_{N, s} \int_{K}|x|^{-2 s}|\hat{\psi}(x)|^{2} d x .
\end{aligned}
$$

If, by contradiction, $\operatorname{Cap}_{\mathbb{R}^{N}}^{s}(K, \hat{\psi})=0$ the above inequality would imply $\hat{\psi}=0$ a.e. in $K$. Since the $N$-dimensional Lebesgue measure of $K$ is strictly positive and $\hat{\psi}$ weakly solves $(-\Delta)^{s} \hat{\psi}=0$ in $\mathbb{R}^{N}$, the Unique Continuation Principle from sets of positive measure proved in [15. Theorem 1.4] would imply that $\hat{\psi} \equiv 0$ in $\mathbb{R}^{N}$, giving rise to a contradiction in view of Remark 2.2. 


\section{Appendix A. Boundedness of Eigenfunctions}

To prove boundedness of eigenfunctions we need the following Sobolev-trace inequality which follows from combination of Theorem 2.3 (i) and continuity of the trace map (2.1) (see also 6, Theorem 2.1]): there exists a positive constant $\tau_{N, s}>0$ such that

$$
\tau_{N, s}\|\operatorname{Tr} W\|_{L^{2^{*}(s)}\left(\mathbb{R}^{N}\right)}^{2} \leq \int_{\mathbb{R}_{+}^{N+1}} t^{1-2 s}|\nabla W|^{2} d t d x, \quad \text { for all } W \in \mathcal{D}^{1,2}\left(\mathbb{R}_{+}^{N+1} ; t^{1-2 s}\right),
$$

where $2^{*}(s)$ is defined in (2.11). In the following lemma we prove that the extensions of eigenfunctions of $(1.3)$ are bounded in $\mathbb{R}_{+}^{N+1}$.

Lemma A.1. Let $\Omega \subset \mathbb{R}^{N}, N \geq 1$, be a bounded open set and $s \in(0, \min \{1, N / 2\})$. Let $\alpha \in \mathbb{R}$ and $W \in \mathcal{D}_{\Omega^{c}}^{1,2}\left(\mathbb{R}_{+}^{N+1} ; t^{1-2 s}\right)$ be a weak solution to

$$
\begin{cases}-\operatorname{div}\left(t^{1-2 s} \nabla W\right)=0, & \text { in } \mathbb{R}_{+}^{N+1}, \\ \lim _{t \rightarrow 0^{+}}\left(-t^{1-2 s} \partial_{t} W\right)=\alpha W, & \text { in } \Omega \times\{0\}, \\ W=0, & \text { in }\left(\mathbb{R}^{N} \backslash \Omega\right) \times\{0\},\end{cases}
$$

in the sense that

$$
\int_{\mathbb{R}_{+}^{N+1}} t^{1-2 s} \nabla W \cdot \nabla \phi d x d t=\alpha \int_{\Omega} \operatorname{Tr} W \operatorname{Tr} \phi d x
$$

for every $\phi \in \mathcal{D}_{\Omega^{c}}^{1,2}\left(\mathbb{R}_{+}^{N+1} ; t^{1-2 s}\right)$. Then $W \in L^{\infty}\left(\mathbb{R}_{+}^{N+1}\right)$ and $\operatorname{Tr} W \in L^{\infty}(\Omega)$.

Proof. The fact that $\operatorname{Tr} W \in L^{\infty}(\Omega)$ can be found in 9 , Theorem 3.1, Remark 3.2], see also [17. Let us prove the statement about its extension. From the Poisson formula for problem (A.2) given in 11] we have that, for some constant $C_{N, s}$,

$$
W(x, t)=C_{N, s} \int_{\mathbb{R}^{N}} \frac{t^{2 s}}{\left(|x-\xi|^{2}+t^{2}\right)^{\frac{N+2 s}{2}}} \operatorname{Tr}(W)(\xi) d \xi \quad \text { for all }(x, t) \in \mathbb{R}_{+}^{N+1},
$$

hence

$$
\begin{aligned}
|W(x, t)| & \leq\|\operatorname{Tr} W\|_{L^{\infty}(\Omega)}\left|C_{N, s}\right| \int_{\mathbb{R}^{N}} \frac{t^{2 s}}{\left(|x-\xi|^{2}+t^{2}\right)^{\frac{N+2 s}{2}}} d \xi \\
& =\|\operatorname{Tr} W\|_{L^{\infty}(\Omega)}\left|C_{N, s}\right| \int_{\mathbb{R}^{N}} \frac{t^{2 s}}{\left(|\xi|^{2}+t^{2}\right)^{\frac{N+2 s}{2}}} d \xi \\
& =\|\operatorname{Tr} W\|_{L^{\infty}(\Omega)}\left|C_{N, s}\right| \int_{\mathbb{R}^{N}} \frac{d \xi^{\prime}}{\left(\left|\xi^{\prime}\right|^{2}+1\right)^{\frac{N+2 s}{2}}} d \xi^{\prime}
\end{aligned}
$$

for all $(x, t) \in \mathbb{R}_{+}^{N+1}$, thus implying that $W \in L^{\infty}\left(\mathbb{R}_{+}^{N+1}\right)$ and completing the proof.

Appendix B. Fractional convergence of Sets in the sense of Mosco

We give the following definition which is the analogue of the standard sets convergence in the sense of Mosco (23).

Definition B.1. Let $\Omega \subset \mathbb{R}^{N}$ be a bounded open set. Let $\left\{K_{\varepsilon}\right\}_{\varepsilon>0}$ be a family of compact sets contained in $\Omega$. We say that $\left\{\Omega \backslash K_{\varepsilon}\right\}_{\varepsilon>0}$ converges to $\Omega \backslash K$ in the fractional sense of Mosco if the following two properties hold:

(i) the weak limit points in $\mathcal{D}^{s, 2}\left(\mathbb{R}^{N}\right)$ of every family of functions $u_{\varepsilon} \in \mathcal{D}^{s, 2}\left(\Omega \backslash K_{\varepsilon}\right)$ belong to $\mathcal{D}^{s, 2}(\Omega \backslash K)$

(ii) for every $u \in \mathcal{D}^{s, 2}(\Omega \backslash K)$, there exists a family of functions $u_{\varepsilon} \in \mathcal{D}^{s, 2}\left(\Omega \backslash K_{\varepsilon}\right)$ such that $u_{\varepsilon} \rightarrow u$ in $\mathcal{D}^{s, 2}\left(\mathbb{R}^{N}\right)$.

In this appendix we prove that the notion of concentration introduced in Definition 1.3 implies the convergence of $\Omega \backslash K_{\varepsilon}$ to $\Omega \backslash K$ in the fractional sense of Mosco if $\operatorname{Cap}_{\Omega}^{s}(K)=0$. 
Lemma B.2. Let $\Omega \subset \mathbb{R}^{N}$ be a bounded open set and $K \subset \Omega$ be a compact set with $\operatorname{Cap}_{\Omega}^{s}(K)=$ 0 . Let $\left\{K_{\varepsilon}\right\}_{\varepsilon>0}$ be a family of compact sets contained in $\Omega$ concentrating to $K$ in the sense of Definition 1.3. Then $\Omega \backslash K_{\varepsilon}$ converges to $\Omega \backslash K$ in the fractional sense of Mosco as $\varepsilon \rightarrow 0^{+}$.

Proof. We first prove that condition (i) in Definition B.1 is satisfied. Let us consider a family $\left\{u_{\varepsilon}\right\}_{\varepsilon>0} \subset \mathcal{D}^{s, 2}\left(\Omega \backslash K_{\varepsilon}\right)$ such that $u_{\varepsilon} \rightarrow u$ in $\mathcal{D}^{s, 2}\left(\mathbb{R}^{N}\right)$. We need to show that $u \in \mathcal{D}^{s, 2}(\Omega \backslash K)$. Obviously $\left\{u_{\varepsilon}\right\}_{\varepsilon>0} \subset \mathcal{D}^{s, 2}(\Omega)$ and $\mathcal{D}^{s, 2}(\Omega)$ is a closed subspace of $\mathcal{D}^{s, 2}\left(\mathbb{R}^{N}\right)$. Then $u \in \mathcal{D}^{s, 2}(\Omega)$ since this space is closed in the weak topology. Furthermore, being $\operatorname{Cap}_{\Omega}^{s}(K)=0$, Proposition 2.6 provides $\mathcal{D}^{s, 2}(\Omega)=\mathcal{D}^{s, 2}(\Omega \backslash K)$.

We now address item (ii) in Definition B.1. Let $u \in \mathcal{D}^{s, 2}(\Omega \backslash K)$ and $U=\mathcal{H}(u)$ be its CaffarelliSilvestre extension as in (2.2). We need to exhibit a sequence $u_{\varepsilon}$ in $\mathcal{D}^{s, 2}\left(\Omega \backslash K_{\varepsilon}\right)$ which converges to $u$ in $\mathcal{D}^{s, 2}\left(\mathbb{R}^{N}\right)$. We note that for every $\delta>0$ there exists $\varphi_{\delta} \in C_{c}^{\infty}\left(\mathbb{R}_{+}^{N+1} \cup \Omega\right)$ such that

$$
\left\|\varphi_{\delta}-U\right\|_{\mathcal{D}^{1,2}\left(\mathbb{R}_{+}^{N+1} ; t^{1-2 s}\right)}<\delta
$$

Since by assumption $\operatorname{Cap}_{\Omega}^{s}(K)=0$, then for every $n \in \mathbb{N}$ there exists $\varepsilon_{n}>0$ and $\eta_{n} \in C_{c}^{\infty}\left(\overline{\mathbb{R}_{+}^{N+1}}\right)$ such that $\left\{\varepsilon_{n}\right\}$ is strictly decreasing to zero, $\eta_{n} \equiv 0$ in $\mathbb{R}^{N} \backslash \Omega, \eta_{n} \equiv 1$ in a neighborhood of $K_{\varepsilon}$ for all $\varepsilon \in\left(0, \varepsilon_{n}\right)$ and

$$
\int_{\mathbb{R}_{+}^{N+1}} t^{1-2 s}\left|\nabla \eta_{n}\right|^{2} d x d t<\frac{1}{n} .
$$

Let us define $W_{n}:=\varphi_{\delta}\left(1-\eta_{n}\right)$. We note that $W_{n} \in \mathcal{D}_{\Omega^{c} \cup K_{\varepsilon}}^{1,2}\left(\mathbb{R}_{+}^{N+1} ; t^{1-2 s}\right)$ for all $\varepsilon \in\left(0, \varepsilon_{n}\right)$. Then, using (2.15) we obtain

$$
\begin{aligned}
\int_{\mathbb{R}_{+}^{N+1}} t^{1-2 s} & \left|\nabla W_{n}-\nabla \varphi_{\delta}\right|^{2} d x d t=\int_{\mathbb{R}_{+}^{N+1}} t^{1-2 s}\left|\nabla\left(\varphi_{\delta} \eta_{n}\right)\right|^{2} d x d t \\
& \leq 2 \int_{\mathbb{R}_{+}^{N+1}} t^{1-2 s}\left|\varphi_{\delta}\right|^{2}\left|\nabla \eta_{n}\right|^{2} d x d t+2 \int_{\mathbb{R}_{+}^{N+1}} t^{1-2 s}\left|\eta_{n}\right|^{2}\left|\nabla \varphi_{\delta}\right|^{2} d x d t \\
& \leq \frac{2 \sup \left|\varphi_{\delta}\right|^{2}}{n}+2\left(\sup \left|\nabla \varphi_{\delta}\right|^{2}\right)\left(\sup _{z \in \operatorname{supp} \varphi_{\delta}}|z|^{2}\right) \int_{\mathbb{R}_{+}^{N+1}} t^{1-2 s} \frac{\left|\eta_{n}\right|^{2}}{|x|^{2}+t^{2}} d x d t \\
& \leq \frac{2 \sup \left|\varphi_{\delta}\right|^{2}}{n}+\frac{8}{n(N-2 s)^{2}}\left(\sup \left|\nabla \varphi_{\delta}\right|^{2}\right)\left(\sup _{z \in \operatorname{supp} \varphi_{\delta}}|z|^{2}\right) .
\end{aligned}
$$

Hence there exists $n_{\delta}$ such that

$$
\left\|W_{n}-\varphi_{\delta}\right\|_{\mathcal{D}^{1,2}\left(\mathbb{R}_{+}^{N+1} ; t^{1-2 s}\right)}<\delta \quad \text { for all } n \geq n_{\delta} .
$$

For all $\varepsilon \in\left(0, \varepsilon_{1}\right)$ we let $U_{\varepsilon}:=W_{n}$ where $n$ is such that $\varepsilon_{n+1} \leq \varepsilon<\varepsilon_{n}$. The above argument then yields that $U_{\varepsilon} \in \mathcal{D}_{\Omega^{c} \cup K_{\varepsilon}}^{1,2}\left(\mathbb{R}_{+}^{N+1} ; t^{1-2 s}\right)$ and

$$
\left\|U_{\varepsilon}-\varphi_{\delta}\right\|_{\mathcal{D}^{1,2}\left(\mathbb{R}_{+}^{N+1} ; t^{1-2 s}\right)}<\delta \quad \text { for all } \varepsilon \in\left(0, \varepsilon_{n_{\delta}}\right) .
$$

Hence $\left\|U_{\varepsilon}-U\right\|_{\mathcal{D}^{1,2}\left(\mathbb{R}_{+}^{N+1} ; t^{1-2 s}\right)} \leq\left\|U_{\varepsilon}-\varphi_{\delta}\right\|_{\mathcal{D}^{1,2}\left(\mathbb{R}_{+}^{N+1} ; t^{1-2 s}\right)}+\left\|\varphi_{\delta}-U\right\|_{\mathcal{D}^{1,2}\left(\mathbb{R}_{+}^{N+1} ; t^{1-2 s}\right)}<2 \delta$ for all $\varepsilon \in\left(0, \varepsilon_{n_{\delta}}\right)$.

We conclude that $U_{\varepsilon} \rightarrow U$ in $\mathcal{D}^{1,2}\left(\mathbb{R}_{+}^{N+1} ; t^{1-2 s}\right)$ and therefore $u_{\varepsilon}=\operatorname{Tr} U_{\varepsilon} \in \mathcal{D}^{s, 2}\left(\Omega \backslash K_{\varepsilon}\right)$ converges to $u=\operatorname{Tr} U$ in $\mathcal{D}^{s, 2}\left(\mathbb{R}^{N}\right)$ by continuity of the trace map (2.1).

\section{REFERENCES}

[1] L. Abatangelo, V. Felli, L. Hillairet, and C. Lena. Spectral stability under removal of small capacity sets and applications to aharonov-bohm operators. Journal of Spectral Theory (online first), DOI 10.4171/JST/251.

[2] D. R. Adams. Besov capacity redux. J. Math. Sci. (N.Y.), 162(3):307-318, 2009. Problems in mathematical analysis. No. 42 .

[3] D. R. Adams and J. Xiao. Strong type estimates for homogeneous Besov capacities. Math. Ann., 325(4):695$709,2003$.

[4] J. Bertrand and B. Colbois. Capacité et inégalité de Faber-Krahn dans $\mathbb{R}^{n}$. J. Funct. Anal., 232(1):1-28, 2006.

[5] G. Besson. Comportement asymptotique des valeurs propres du laplacien dans un domaine avec un trou. Bull. Soc. Math. France, 113(2):211-230, 1985. 
[6] C. Brändle, E. Colorado, A. de Pablo, and U. Sánchez. A concave-convex elliptic problem involving the fractional laplacian. Proceedings of the Royal Society of Edinburgh Section A: Mathematics, 143(1):39-71, 2013.

[7] L. Brasco and E. Cinti. On fractional Hardy inequalities in convex sets. Discrete Contin. Dyn. Syst., to appear.

[8] L. Brasco, E. Lindgren, and E. Parini. The fractional Cheeger problem. Interfaces Free Bound., 16(3):419-458, 2014.

[9] L. Brasco and E. Parini. The second eigenvalue of the fractional p-Laplacian. Adv. Calc. Var., 9(4):323-355, 2016.

[10] L. Brasco and A. Salort. A note on homogeneous Sobolev spaces of fractional order. Preprint 2018.

[11] L. Caffarelli and L. Silvestre. An extension problem related to the fractional Laplacian. Comm. Partial Differential Equations, 32(7-9):1245-1260, 2007.

[12] I. Chavel and E. A. Feldman. Spectra of manifolds less a small domain. Duke Math. J., 56(2):399-414, 1988.

[13] G. Courtois. Spectrum of manifolds with holes. J. Funct. Anal., 134(1):194-221, 1995.

[14] E. Di Nezza, G. Palatucci, and E. Valdinoci. Hitchhiker's guide to the fractional Sobolev spaces. Bull. Sci. Math., 136(5):521-573, 2012.

[15] M. M. Fall and V. Felli. Unique continuation property and local asymptotics of solutions to fractional elliptic equations. Communications in Partial Differential Equations, 39(2):354-397, 2014.

[16] M. Flucher. Approximation of Dirichlet eigenvalues on domains with small holes. J. Math. Anal. Appl., 193(1):169-199, 1995.

[17] G. Franzina and G. Palatucci. Fractional p-eigenvalues. Riv. Math. Univ. Parma (N.S.), 5(2):373-386, 2014.

[18] N. Garofalo and F.-H. Lin. Monotonicity properties of variational integrals, $A_{p}$ weights and unique continuation. Indiana Univ. Math. J., 35(2):245-268, 1986.

[19] P. Grisvard. Elliptic problems in nonsmooth domains, volume 69 of Classics in Applied Mathematics. Society for Industrial and Applied Mathematics (SIAM), Philadelphia, PA, 2011. Reprint of the 1985 original [ MR0775683], With a foreword by Susanne C. Brenner.

[20] J. Heinonen, T. Kilpeläinen, and O. Martio. Nonlinear potential theory of degenerate elliptic equations. Courier Corporation, 2012.

[21] B. Helffer. Spectral theory and its applications, volume 139. Cambridge University Press, 2013.

[22] I. W. Herbst. Spectral theory of the operator $\left(p^{2}+m^{2}\right)^{1 / 2}-Z e^{2} / r$. Comm. Math. Phys., 53(3):285-294, 1977.

[23] U. Mosco. Convergence of convex sets and of solutions of variational inequalities. Advances in Mathematics, 3(4):510-585, 1969.

[24] R. Musina and A. I. Nazarov. On fractional Laplacians. Comm. Partial Differential Equations, 39(9):1780$1790,2014$.

[25] S. Ozawa. Singular variation of domains and eigenvalues of the Laplacian. Duke Math. J., 48(4):767-778, 1981.

[26] J. Rauch and M. Taylor. Potential and scattering theory on wildly perturbed domains. J. Funct. Anal., 18:2759, 1975.

[27] A. Ritorto. Optimal partition problems for the fractional Laplacian. Ann. Mat. Pura Appl. (4), 197(2):501-516, 2018.

[28] A. Rüland. Unique continuation for fractional Schrödinger equations with rough potentials. Comm. Partial Differential Equations, 40(1):77-114, 2015.

[29] R. Servadei and E. Valdinoci. On the spectrum of two different fractional operators. Proc. Roy. Soc. Edinburgh Sect. A, 144(4):831-855, 2014.

[30] S. Shi and J. Xiao. On fractional capacities relative to bounded open Lipschitz sets. Potential Anal., 45(2):261298, 2016.

[31] X. Tao and S. Zhang. Boundary unique continuation theorems under zero Neumann boundary conditions. Bull. Austral. Math. Soc., 72(1):67-85, 2005.

[32] M. Warma. The fractional relative capacity and the fractional Laplacian with Neumann and Robin boundary conditions on open sets. Potential Anal., 42(2):499-547, 2015.

Laura Abatangelo

Dipartimento di Matematica e Applicazioni, Università degli Studi di Milano-Bicocca,

Via Cozzi 55, 20125 Milano, Italy.

E-mail address: laura.abatangelo@unimib.it

VERONICA FELLI

Dipartimento di Scienza dei Materiali, Università degli Studi di Milano-Bicocca,

Via Cozzi 55, 20125 Milano, Italy.

E-mail address: veronica.felli@unimib.it

Benedetta Noris

LAMFA: Laboratoire Amiénois de Mathématique Fondamentale et Appliquée, UPJV Université de Picardie Jules Verne, 33 rue Saint-Leu, 80039 Amiens, France.

E-mail address: benedetta.noris@u-picardie.fr 